\title{
Unexpected Insights about Cation-Exchange on Metal Oxide Nanoparticles and Its Effect on Their Magnetic Behavior
}

\author{
Sergio Lentijo-Mozo, ${ }^{\dagger}$ Davide Deiana, ${ }^{\dagger}$ Elisa Sogne, ${ }^{\dagger}$ Alberto Casu, ${ }^{*}{ }^{\dagger}$ and Andrea Falqui ${ }^{*},^{\dagger}$ (i) \\ ${ }^{\dagger}$ King Abdullah University of Science and Technology (KAUST), Biological and Environmental Sciences and Engineering (BESE) \\ Division, Nabla Lab, Thuwal 23955-6900, Saudi Arabia \\ ${ }^{\ddagger}$ Centre Interdisciplinaire de Microscopie Électronique (CIME), Ecole Polytechnique Fédérale de Lausanne (EPFL), 1015 \\ Lausanne, Switzerland
}

\section{Supporting Information}

\begin{abstract}
Tuning the magnetic behavior of nanoparticles via the control of their features has always been challenging because these features are mostly intertwined. In the last years, a novel synthetic approach based on cation-exchange has been reported, and one of its main advantages is to maintain the shape and size of nanoparticles. However, such a synthetic strategy has been seldom applied to iron oxide magnetic nanoparticles, where the substitution of iron with diverse transition element cations was described as occurring in their whole volume. Surprisingly, we found results quite discordant from the few ones so far published in exploiting again this approach. We show here that it unavoidably leads to core/shell structures with only the shell undergoing the cation-exchange.

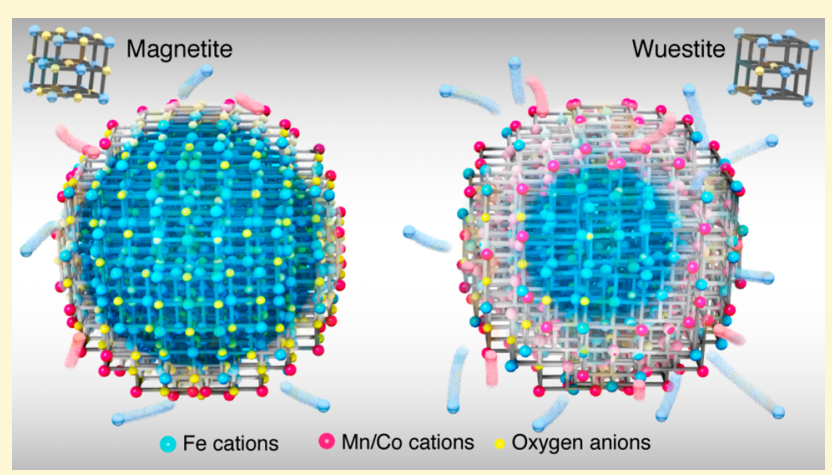
Moreover, the starting phase of iron oxide strongly dictates the number of iron cations that could be replaced: if it is structurally free of vacancies, like magnetite, the maximum amount of exchanged cations is low and only affects the nanoparticles' most external, disordered layers. Conversely, the cation-exchange is boosted if the iron oxide phase is structurally prone to vacancies, like wüstite, and the shell where the iron cations have been partly substituted becomes quite thicker. These findings are further corroborated by the materials' magnetic properties.
\end{abstract}

\section{INTRODUCTION}

The possibility to tune the magnetic properties of nanoparticles (NPs) by controlling their morphology (i.e., shape, mean size, and distribution), crystal structure, chemical composition, and level of mutual interaction is a well-known field of research because of its fundamental and applicative implications. Here, the strong interdependence between compositional and morphostructural parameters implies that any improvement of the magnetic response is ascribed to a joint effect involving all the NPs features, rather than to the modification of single parameters. ${ }^{1-5}$ In this regard, the higher degree of control over the NPs morphology granted by different synthetic routes represents a step forward to limit the number of free intertwined parameters. Cation-exchange (CE) has been largely shown to be capable of providing a significant advantage in changing the composition and possibly the related crystal structure of diverse nanosized crystals, given its capacity to partially or totally substitute the NPs cations without affecting their initial shape and size. This strategy revealed itself to be very effective when applied to chalcogenides nanosized crystals, ${ }^{6-13}$ even using an in situ transmission electron microscopy (TEM) approach at the solid state developed by our group. ${ }^{14-16}$ Also, it has been clearly shown that, for such nanocrystals, the amount of exchanged cations can be strongly boosted by increasing the number of available vacancies in the crystal structure of the compound undergoing the $\mathrm{CE}$ reaction. ${ }^{17}$ Because of its peculiar and promising features, CE has then been applied to magnetic metal oxides with the aim to get an unprecedentedly fine way for tuning the magnetic behavior of a given NPs population, via the precise control and subsequent modification of its compositional features. ${ }^{18,19}$

More in detail, in 2013 Sytnyk et al. published a paper dealing with $\mathrm{CE}$ as an effective route to change the composition of iron oxide-based magnetic nanoparticles, keeping their shape and size unaltered. ${ }^{18}$ Such an approach was applied to three different kinds of nanoparticles, namely, magnetite $\left(\mathrm{Fe}_{3} \mathrm{O}_{4}\right)$ spherical nanoparticles, gold/magnetite, and wüstite $\left(\mathrm{Fe}_{x} \mathrm{O}\right) /$ cobalt ferrite core/shell nanoparticles, respectively. This work showed that a cation exchange approach could be successfully exploited to tune the nanoparticles magnetic properties via their compositional modification, in that case partially exchanging iron cations $\left(\mathrm{Fe}^{2+}\right)$ with cobalt ones $\left(\mathrm{Co}^{2+}\right)$. However, even if in that paper

Received: October 11, 2018

Revised: October 21, 2018

Published: October 22, 2018 
the iron $\rightarrow$ cobalt $\mathrm{CE}$ was indicated as occurring over the whole volume of the iron oxide NPs, the there-reported Co compositional profiles actually looked much more likely ascribable to a nonuniform localization of the exchanging element in the particles volume, giving rise to core/shell structures. In this regard, a possible study of the hysteresis loop of the sample after cooling it in a magnetic field of sufficient strength would have greatly clarified this point, because in the case of a core/shell structure it would have likely shown a horizontal shift of the hysteresis loops due to the magnetic coupling, also called exchange-bias (EB), of core and shell via their interface. ${ }^{20-24}$ Unfortunately, as the authors classified the cobalt as homogeneously distributed over the whole body of the particles, that measurement was not taken into account. Furthermore, something interesting occurred in the wüstite/ magnetite core/shell nanoparticles also reported in the paper of Sytnyk et al.: ${ }^{18}$ at a deeper look, the percentage of cobalt contained in the shell is lower than that expected in the case of a stoichiometric cobalt ferrite, pointing out a further puzzling point that will be discussed in greater detail in the Results and Discussion section. A synthetic approach similar to the one adopted by Sytnyk et al. ${ }^{18}$ was more recently published by Zhao et al., ${ }^{19}$ where magnetite nanoparticles were submitted to $\mathrm{CE}$ in order to partially substitute the iron cations with either $\mathrm{Mn}$ or $\mathrm{Zn}$. Since the main aim of this latter work was making the particles more effective as contrast agents for in vivo magnetic resonance imaging, the reported compositional characterization of the exchanged particles is basic: no compositional profile of the NPs is shown, the displayed EDS maps are quite poor in terms of statistics and any reference image of the particles mapped by EDS is missing. Indeed, no determination of the $\mathrm{Mn}$ and $\mathrm{Zn} \mathrm{At \%}$ is derived from the EDS spectra corresponding to the maps. On the other hand, it is also noteworthy citing two further works that very recently showed that changing the composition of transition metal oxides magnetic nanoparticles via CE could result in the formation of core/shell structures. ${ }^{25,26}$ In the first one, published by Luo et al., the authors showed that by performing a CE reaction on $\mathrm{Mn}_{3} \mathrm{O}_{4}$ nanoparticles they first succeeded in transforming the external layer of these particles in a shell of $\mathrm{CoMn}_{2} \mathrm{O}_{4}$ while keeping a $\mathrm{Mn}_{3} \mathrm{O}_{4}$ core and with just minimal shape change. Moreover, changing both the Co precursor and its concentration, a further discontinuous shell of cobalt oxide was overgrown on the $\mathrm{Mn}_{3} \mathrm{O}_{4} / \mathrm{CoMn}_{2} \mathrm{O}_{4}$ core/shell NPs. ${ }^{25}$ Something quite similar was found also by our group: making use of a CE approach, we were able to prepare from $\mathrm{Fe}_{3} \mathrm{O}_{4}$ / $\mathrm{MnFe}_{2} \mathrm{O}_{4}$ core/single shell to $\mathrm{Fe}_{3} \mathrm{O}_{4} / \mathrm{MnFe}_{2} \mathrm{O}_{4} / \mathrm{Mn}_{3} \mathrm{O}_{4}$ core/ double shell particles, by just properly tuning the amount of Mn precursor. ${ }^{26}$

All these findings first pushed us to follow the same synthetic route reported by Sytnyk et al., ${ }^{18}$ with the aim of studying much more deeply the localization of the exchanging cation species in the particles. We used cobalt and manganese to exchange iron in pure magnetite and pure wüstite nanoparticles, focusing our attention on the possible influence of crystal structure toward the outcome of CE. By carefully tuning the morphology of both NPs populations, we obtained parallel sets of monodispersed, morphologically equivalent but structurally different NPs. The results of our studies first show minimal morphological differences between the NPs before and after CE, as long as the amount of the substituting ion's precursor is kept much lower than that reported in ref 18 . Furthermore, repeating the same CE reactions on intrinsically undefective $\left(\mathrm{Fe}_{3} \mathrm{O}_{4}\right)$ and defective $\left(\mathrm{Fe}_{x} \mathrm{O}\right)$ NPs and comparing the initial and final steps of both sets of reactions through indepth electron microscopy and magnetic studies, we find that the exchanged NPs always display a core/shell structure, as clearly highlighted by the compositional profiles of the substituting ions, which are actually similar to those reported in the work of Sytnyk et al. In fact, the corresponding elemental maps clearly indicate that both cobalt and manganese are much more localized in the external part of the particles, giving rise to actual core/shell NPs. If the same $\mathrm{CE}$ reaction is applied to either undefective $\mathrm{Fe}_{3} \mathrm{O}_{4}$ or defective $\mathrm{Fe}_{x} \mathrm{O}$ NPs (both sized around $10 \mathrm{~nm}$ ), the amount of exchanged cobalt and manganese found in the particles strongly changes, going from 3 to $4 \mathrm{At} \%$ for $\mathrm{Fe}_{3} \mathrm{O}_{4}$ to more than $20 \%$ for $\mathrm{Fe}_{x} \mathrm{O}$. Thus, excluding morphology-related major variations during the reactions as size and shape of the starting iron oxide NPs were carefully maintained in all cases, the different outcomes of $\mathrm{CE}$ reactions can be ascribed to the only parameter that differentiated the NPs (i.e., their crystal phase). Furthermore, we find that the presence of a thicker cationexchanged shell in the $\mathrm{Fe}_{x} \mathrm{O}$-based NPs has to be ascribed to the intrinsic defectivity of the wüstite matrix, which makes the NPs more prone to $\mathrm{CE}$, while analogous $\mathrm{CE}$ reactions conducted on $\mathrm{Fe}_{3} \mathrm{O}_{4}$ NPs lead to a thinner cation-exchanged shell. In turn, these findings provide a solid foundation to explain the magnetic features of both populations and their evolution after CE. The different crystal structure accounts for both the high magnetic hardness of the initial antiferromagnetic $\mathrm{Fe}_{x} \mathrm{O}$ NPs with respect to $\mathrm{Fe}_{3} \mathrm{O}_{4}$ and the effects of $\mathrm{CE}$ (i.e., a magnetic softening/hardening after $\mathrm{CE}$ by $\mathrm{Mn} / \mathrm{Co}$, which is more pronounced for the thick-shelled $\mathrm{Fe}_{x} \mathrm{O}$ exchanged NPs). Finally, we show how all our samples, both the $10 \mathrm{~nm}$ sized magnetite-based and $9 \mathrm{~nm}$ sized wüstite-based NPs, show spin-glass-like features at low temperature, both before and after the cation-exchange reactions.

\section{EXPERIMENTAL SECTION}

Materials. Oleic acid (OA, 99\%), oleylamine (OLA, 70\%), 1octadecene (90\%), trioctylamine (98\%), octylether (99\%), the manganese precursor $\mathrm{MnCl}_{2}$ (99\%), and the iron precursors: $\mathrm{FeCl}_{3}$. $6 \mathrm{H}_{2} \mathrm{O}$ (98\%), $\mathrm{Fe}(\mathrm{CO})_{5}$ (99.99\%) were purchased from SigmaAldrich. The cobalt precursor $\mathrm{CoCl}_{2}$ (99\%) was purchased from Fisher-U.S. Sodium oleate (97\%) was purchased by TCI, and trioctylphospine (TOP, 97\%) was purchased from Strem chemicals. Organic solvents like acetone, ethanol, and hexane were of analytical grade and obtained from various sources. Anhydrous THF (99.9\%) and anhydrous methanol (99.8\%) were purchased from Aldrich. All chemicals were used as received without any further purification. All experiments were carried out using standard airless techniques: a vacuum/dry nitrogen gas Schlenk line was used for synthesis and an Ar glovebox for storing and handling air- and moisture-sensitive chemicals.

Preparation of the Iron(III)-Oleate Precursor. $\mathrm{FeCl}_{3} \cdot 6 \mathrm{H}_{2} \mathrm{O}$ and sodium oleate were dissolved in a 1.5:1:2.5 ratio solution of ethanol, deionized water, and hexane. The resulting solution was heated at 70 ${ }^{\circ} \mathrm{C}$ for $4 \mathrm{~h}$. When the reaction was complete, the brown mixture was transferred to a separatory funnel, and the aqueous layer was removed. The organic phase that contains the iron-oleate complex was washed three times with deionized water. After the brown solution was washed, it was dried with $\mathrm{Na}_{2} \mathrm{SO}_{4}$ anhydrous, and hexane was removed by evaporation. The final product was dried under vacuum to obtain a brown waxy solid.

Synthesis of Magnetite Spherical NPs with a Diameter of $21 \mathrm{~nm}$. A solution containing iron oleate in trioctylamine and oleic acid (molar ratio iron oleate to oleic acid was 1:0.5) were mixed in a three-neck round-bottom flask at room temperature. The reaction 
solution was heated to $120{ }^{\circ} \mathrm{C}$ and degassed under vacuum for 30 min. After being filled with a nitrogen atmosphere, the mixture reaction was heated up to $360{ }^{\circ} \mathrm{C}$ with a heating rate about $3.3{ }^{\circ} \mathrm{C} /$ $\mathrm{min}$ and aged for $30 \mathrm{~min}$. The resulting black mixture was cooled to room temperature, and acetone was added to precipitate the NPs. They were separated by centrifugation and washed with acetone several times until the supernatant was colorless. After they were washed, the NPs were dried and stored as a waxy solid. Their mean sizes were measured by conventional TEM and found to be $21.4 \pm$ $1.3 \mathrm{~nm}$. This sample will be named M21.

Synthesis of Magnetite Spherical NPs with a Diameter of $10 \mathrm{~nm}$. A solution containing iron oleate in 1-octadecene and oleic acid (molar ratio iron oleate to oleic acid was 1:0.5) were mixed in a three-neck round-bottom flask at room temperature. The reaction solution was heated to $120{ }^{\circ} \mathrm{C}$ and degassed under vacuum for 30 min. After it was filled with a nitrogen atmosphere, the mixture reaction was heated up to $320{ }^{\circ} \mathrm{C}$ with a heating rate about $3.3^{\circ} \mathrm{C} /$ min and aged for $30 \mathrm{~min}$. The resulting black mixture was cooled to room temperature, and acetone was added to precipitate the NPs. They were separated by centrifugation and washed, first with acetone, and second with a mixture of combined solvents hexane/acetone several times until the supernatant was colorless. After washing, the NPs were dried, and stored as a waxy solid. Their mean size were measured by conventional TEM and found being equal to $10.4 \pm 0.9$ $\mathrm{nm}$. This sample will be named M10.

Synthesis of Wüstite Spherical NPs with a Diameter of 9 $\mathrm{nm}$. A solution of oleic acid $(1.1 \mathrm{~g}, 3.8 \mathrm{mmol})$ in octyl ether was prepared in a three-neck round-bottom flask at room temperature. The reaction solution was heated to $100{ }^{\circ} \mathrm{C}$ and degassed under vacuum for $20 \mathrm{~min}$. After being filled with a nitrogen atmosphere, $\mathrm{Fe}(\mathrm{CO})_{5}(1.52 \mathrm{mmol})$ was injected into the solution and the reaction mixture was heated up to reflux at $286{ }^{\circ} \mathrm{C}$. After $10 \mathrm{~min}$, the nucleation started, and the solution became dark. Then, the dark solution was refluxed for an additional $1.5 \mathrm{~h}$. The resulting black mixture was cooled to room temperature, and methanol was added to precipitate the NPs. The solid were separated by centrifugation and washed, first with methanol, and second with a mixture of combined solvents THF/methanol several times until the supernatant was colorless. After the NPs were washed, they were dried and stored as a solid under argon atmosphere. Their mean size was measured by conventional TEM and found to be equal to $9.4 \pm 0.6 \mathrm{~nm}$. This sample will be named W9.

Synthesis of Co-Doped and $\mathrm{Mn}$-Doped $\mathrm{Fe}_{3} \mathrm{O}_{4} \mathrm{NPs}$ with a Diameter of $21 \mathrm{~nm}$ by a Cation Exchange Protocol. A mixture of $\mathrm{CoCl}_{2}(1.02 \mathrm{~g}, 7.9 \mathrm{mmol})$ in OLA was heated at $120^{\circ} \mathrm{C}$ for $20 \mathrm{~min}$ to completely dissolve the $\mathrm{CoCl}_{2}$. Next, $18 \mathrm{mg}$ of M21 sample was dissolved in hexane and then added to the solution under $\mathrm{N}_{2}$ atmosphere, and the reaction mixture was degassed under vacuum to remove the hexane. Then, TOP $(1 \mathrm{~mL})$ was injected under $\mathrm{N}_{2}$ atmosphere, and the reaction mixture was heated to $200{ }^{\circ} \mathrm{C}$ for 30 min. The resulting black mixture was cooled to room temperature (RT), and acetone was added to precipitate the NPs. The solid was separated by centrifugation and washed with acetone several times until the supernatant was colorless. Such a NPs synthesis procedure was repeated twice. First, adding $70 \mathrm{mg}$ of $\mathrm{CoCl}_{2}(0.54 \mathrm{mmol})$, and second, adding $75 \mathrm{mg}$ of $\mathrm{MnCl}_{2}(0.60 \mathrm{mmol})$. In the three cases, the same amount of M21 sample was kept, and the final black solid was dissolved and stored in hexane. The three samples described above were named M21_Co_hc (high content), M21_Co, and M21_Mn, respectively.

Synthesis of $\mathrm{Co} / \mathrm{Mn}$-Doped $\mathrm{Fe}_{3} \mathrm{O}_{4} \mathrm{NPs}$ with a Diameter of $10 \mathrm{~nm}$ by a Cation Exchange Protocol. A mixture of $\mathrm{CoCl}_{2}$ (18 $\mathrm{mg}, 0.14 \mathrm{mmol}$ ) in OLA was heated at $120{ }^{\circ} \mathrm{C}$ for $20 \mathrm{~min}$ to completely dissolve the $\mathrm{CoCl}_{2}$. After that, $17 \mathrm{mg}$ of M10 sample dissolved in hexane were added to the solution under $\mathrm{N}_{2}$ atmosphere, and the reaction mixture was degassed under vacuum to remove the hexane. Then, TOP $(1 \mathrm{~mL})$ was injected under $\mathrm{N}_{2}$ atmosphere, and the reaction mixture was heated to $200{ }^{\circ} \mathrm{C}$ for $30 \mathrm{~min}$. The resulting black mixture was cooled to room temperature (RT), and acetone was added to precipitate the NPs. The solid was separated by centrifugation and washed with acetone several times until the supernatant was colorless. Such a NPs synthesis procedure was repeated once, adding $18 \mathrm{mg}$ of $\mathrm{MnCl}_{2}(0.14 \mathrm{mmol})$. In the two cases, the same amount of M10 sample was kept, and the final black solid was dissolved and stored in hexane. The two samples, of which the synthesis is described above, will be named M10_Co and M10_Mn, respectively.

Synthesis of $\mathrm{Co} / \mathrm{Mn}$-Doped $\mathrm{Fe}_{x} \mathrm{O}$ NPs with a Diameter of 9 $\mathrm{nm}$ by a Cation Exchange Protocol. A mixture of $\mathrm{CoCl}_{2}(52 \mathrm{mg}$, $0.4 \mathrm{mmol}$ ) in OLA was heated at $120^{\circ} \mathrm{C}$ for $20 \mathrm{~min}$ to completely dissolve the $\mathrm{CoCl}_{2}$. After that, $15 \mathrm{mg}$ of W9 sample dissolved in THF were added to the solution under $\mathrm{N}_{2}$ atmosphere, and the reaction mixture was degassed under vacuum to remove the THF. Then, TOP $(1 \mathrm{~mL})$ was injected under $\mathrm{N}_{2}$ atmosphere, and the reaction mixture was heated to $200{ }^{\circ} \mathrm{C}$ for $30 \mathrm{~min}$. The resulting black mixture was cooled to room temperature (RT), and acetone was added to precipitate the NPs. The solid was separated by centrifugation and washed with acetone several times until the supernatant was colorless. Such a NPs synthesis procedure was repeated once, adding $50 \mathrm{mg}$ of $\mathrm{MnCl}_{2}(0.4 \mathrm{mmol})$. In the two cases, the same amount W9 sample was kept, and the final black solid was dissolved and stored in hexane. The two samples of which the synthesis is described above, will be named W9_Co and W9_Mn, respectively (Table 1).

\section{Table 1. List of Magnetite-Based and Wüstite-Based} Samples

\begin{tabular}{llcc} 
sample & starting material & additional cation & average size (nm) \\
M21 & magnetite & - & 21 \\
M21_Co_hc & magnetite & Co & n.d. \\
M21_Co & magnetite & Co & 21 \\
M21_Mn & magnetite & Mn & 21 \\
M10 & magnetite & - & 10 \\
M10_Co & magnetite & Co & 10 \\
M10_Mn & magnetite & Mn & 10 \\
W9 & wüstite & - & 9 \\
W9_Co & wüstite & Co & 9 \\
W9_Mn & wüstite & Mn & 9 \\
W9ox_Mn & wüstite & Mn & n.d. \\
\hline
\end{tabular}

X-ray Diffraction. X-ray power diffraction (XRD) was performed at room temperature using a Bruker D8 Advance system, equipped with a monochromatic copper radiation source $\mathrm{Cu} \mathrm{K} \alpha=0.154056 \mathrm{~nm}$ in the $30^{\circ}-80^{\circ}$ range with a scan step of $1.6^{\circ} / \mathrm{min}, 0.4^{\circ} / \mathrm{min}$, and $0.07^{\circ} / \mathrm{min}$, respectively.

Transmission Electron Microscopy Characterization. Conventional transmission electron microscopy (TEM) imaging was performed by use of a FEI Tecnai Spirit microscope, equipped with a lanthanum hexaboride thermionic electron source, a Twin objective lens, a Gatan Orius charge-coupled device (CCD) camera, and operating at an acceleration voltage of $120 \mathrm{kV}$. Spherical aberration (Cs) corrected high-resolution and scanning transmission electron microscopy (HRTEM and STEM), along with energy-dispersive Xray spectroscopy (EDS) elemental mapping, were carried out on a double spherical corrected FEI Titan Themis microscope, equipped with an ultrabright Schottky (XFEG) electron source, a SuperX EDS spectrometer with 0.7 srad collection angle, and a FEI Ceta complementary metal oxide semiconductor (CMOS) camera, operating at an acceleration voltage of $200 \mathrm{kV}$. The composition of the core/shell systems was determined with the Cliff-Lorimer method and always averaged over the whole nanoparticle volume. The thickness reported here for the cation-exchanged shells was measured as an average obtained from the line profiles extracted from the EDS maps of multiple particles per sample and taken as the FWHM of the two most intense and external peaks corresponding to the element that gave rise to the cation exchange. Structural characterization was performed by analyzing the 2-Dimensional Fast Fourier Transform 
(2D-FFT) diffractograms and measuring the planar and angular relationships occurring between diffraction spots.

Magnetic Behavior Characterization. Magnetic characterization was performed on a Quantum Design VSM SQUID magnetometer equipped with a superconducting magnet producing fields up to $70 \mathrm{kOe}(7 \mathrm{~T})$ and a Helium Quantum Design Evercool liquefier. Zero-field-cooled (ZFC) and field-cooled (FC) magnetization curves were collected in the $4 \div 300 \mathrm{~K}$ range of temperatures. The magnetic features of magnetite- and wüstite-based pristine nanoparticles and the effect of Fe-to-Mn [Co] CE were studied by DC magnetometry according to the ZFC-FC (zero field cooled-field cooled) and hysteretic loops protocols. The former was adopted to study temperature-dependent variations in magnetization in the $4 \div 300 \mathrm{~K}$ temperature range upon a low applied magnetic field (50 Oe), while the latter studied the isothermal variation in magnetization under a high magnetic field (up to $\pm 70 \mathrm{kOe}$ ) at 4 and $300 \mathrm{~K}$, corresponding to the magnetically blocked and unblocked states. Hysteresis loops were also measured after cooling the samples to $4 \mathrm{~K}$ in strong magnetic field of $70 \mathrm{kOe}$ (FC hysteresis), to highlight the core/shell and superficial disorder effects. The temperature corresponding to the maximum of the ZFC curve and the minimum temperature of superposition between the ZFC and FC curves are indicated as blocking temperature $\left(T_{\mathrm{B}}\right)$ and irreversibility temperature $\left(T_{\mathrm{IRR}}\right)$, respectively, and represent the two main parameters of the ZFC-FC magnetization curves. The two temperatures should tend to the same value for a perfectly monodispersed population of non interacting NPs; thus, the span between $T_{\mathrm{B}}$ and $T_{\text {IRR }}\left(\Delta T=T_{\text {IRR }}-\right.$ $T_{\mathrm{B}}$ ) usually provides a qualitative indication of the distribution in size of the magnetic domains. Saturation magnetization values $\left(M_{\mathrm{S}}\right)$ were determined from the hysteresis loops by extrapolation of $M$ values vs $1 / H$ for $1 / H \rightarrow 0$. The samples were prepared according to a twostep protocol in order to normalize their magnetic response by the effective mass of the magnetic phase. At first, the solutions were dried, and the resulting compounds were measured by thermogravimetric analysis (TGA) to assess the percentage of magnetic phase effectively formed. The dried compounds were then embedded in Teflon tape to prepare the final SQUID sample. All the components of the SQUID samples were weighed for mass normalization. Finally, to check the possible spin-glass like behavior of our samples, we measured their time dependence of thermo-remanent magnetization after cooling them in a field of $50 \mathrm{kOe}$, and recording their magnetization decay at $4 \mathrm{~K}$ after waiting $1200 \mathrm{~s}$ before the field was turned off.

\section{RESULTS AND DISCUSSION}

Our general synthetic strategy required a multiple steps approach, as described in detail in the Experimental Section and summarized as follows. As a first step, we prepared the M21 sample by adapting the two steps-method developed by Park et al. ${ }^{27}$ The TEM and HRTEM images of M21 sample are reported in Figure 1a,d, with the final NPs population being constituted by particles monodispersed in size and spherical in shape, having the crystal structure expected for magnetite.

The M21 NPs were subsequently subjected to the $\mathrm{Fe}^{2+}$ to $\mathrm{Co}^{2+}$ cation exchange according to the synthetic approach described by Sytnyk et al. ${ }^{18}$ In that work, the authors demonstrated that this procedure resulted in a partial extraction of $\mathrm{Fe}^{2+}$ from the initial whole crystal structure of the magnetite $\left(\mathrm{Fe}_{3} \mathrm{O}_{4}\right) \mathrm{NPs}$ and the expected substitution with $\mathrm{Co}^{2+}$ cations, concomitantly preserving size and shape of the starting NPs. Moreover, the authors stated that a cobalt content of $8 \%$ was measured, corresponding to an $x$ mean value of 0.24 calculated on the whole body of the $\mathrm{Co}_{x} \mathrm{Fe}_{3-x} \mathrm{O}_{4}$ spherical NPs they obtained. Despite having carefully reproduced this approach in terms of synthetic route, concentration and amount of chemicals the resulting NPs populations (sample M21_Co_hc) were quite dispersed in size and no longer uniform in chemical composition, and they also
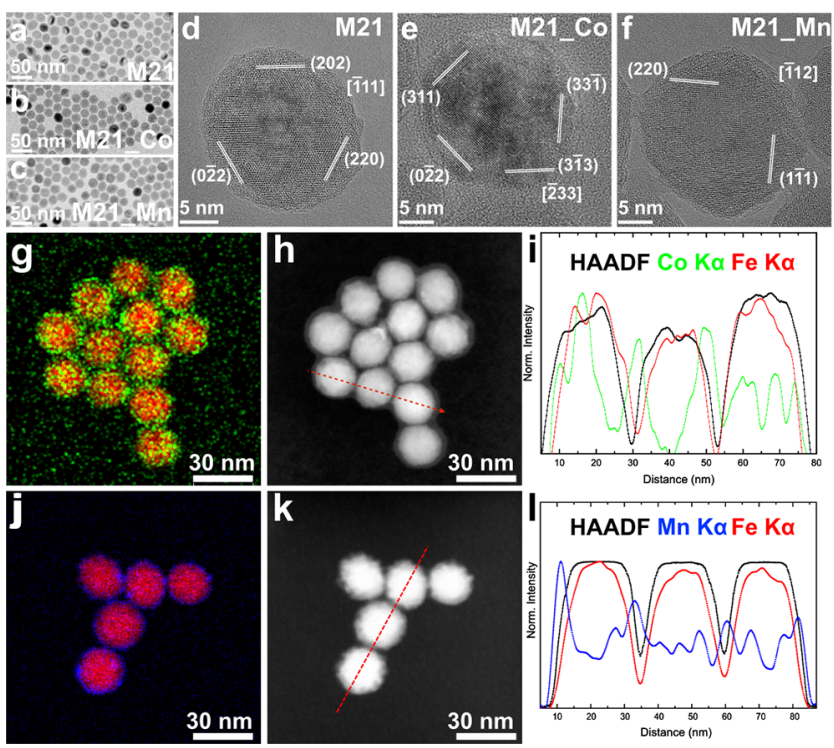

Figure 1. Conventional TEM and HRTEM images of samples M21 (a,d), M21 Co (b,e) and M21 Mn (c,f); STEM-EDS maps, corresponding HAADF-STEM images and compositional profiles of M21 Co $(g-i)$ and M21 Mn $(j-1)$. The same data are also reported as EDS single-element maps in Figure SI_1 for improved readability.

partially lost their initial shape. TEM and HRTEM images, together with STEM/EDS maps and corresponding compositional profiles of M21 Co hc sample are reported in Figure SI_2. As it can be immediately noticed by the spatially resolved EDS compositional mapping, the NPs produced following the $\mathrm{CE}$ approach proposed by Sytnyk et al. brought to two distinct nanoparticles populations: (1) polydisperse core/shell $\mathrm{Fe}_{3} \mathrm{O}_{4}$ / $\mathrm{Co}_{x} \mathrm{Fe}_{3-x} \mathrm{O}_{4}$, with composition averaged over the whole NPs being $x=0.87 \pm 0.05$ and a mean shell thickness of $3.7 \pm 0.9$ $\mathrm{nm}$, and (2) quite larger cobalt oxide particles. We associate the first population to partial dissolution of the starting NPs, caused by the high concentration of cobalt precursor that leads to the loss of monodispersity, together with the formation of a cobalt ferrite shell on top of the resulting $\mathrm{Fe}_{3} \mathrm{O}_{4}$ cores. Moreover, the excess of cobalt precursor is also responsible for the growth of bigger, pure cobalt (oxide) NPs. Although these results look quite different from what described by the paper of Sytnyk et al., it is worth noticing that in that work the shape of the Co compositional profile extracted from the EDS maps, even if visibly binned, clearly recalls the typical M-shaped core/shell profile rather than the dome-shaped one expected for NPs that underwent a uniform CE between iron and cobalt, as extensively reported in the literature. ${ }^{28-31}$

In order to avoid such overtreatment-related effects and to keep the original shape and size of the starting material, we decreased the concentration of the cobalt precursor, while keeping unchanged the sample M21 concentration and other reaction parameters. In this latter synthesis, we chose the highest concentration of cobalt precursor that allowed keeping unaltered the size and shape of the original nanoparticles, as detailed in the Experimental Section. TEM imaging (Figure 1b) of M21 Co sample shows monodispersed spherical NPs with mean diameter $d=22.1 \pm 1.3 \mathrm{~nm}$, thus retaining both shape and size of those constituting the starting M21 sample. HRTEM imaging (Figure 1e) of M21_Co shows that the NPs have monocrystalline structure, but the effect of Co introduction is visible in the STEM-EDS maps and related 
compositional profiles (Figure $1 \mathrm{~g}-\mathrm{i}$ ). The relevant M-shaped profile clearly indicates that cobalt is located in an external $\mathrm{Co}_{x} \mathrm{Fe}_{3-x} \mathrm{O}_{4}$ shell, where $x$ here was measured to be equal to $0.27 \pm 0.05$ and with a mean shell thickness of $3.5 \pm 0.6 \mathrm{~nm}$. The monocrystalline structure observed by HRTEM indicates that the iron-cobalt ion exchange did not change the original magnetite particles structure. This is not unexpected if one considers both the similarity among the magnetite and cobalt ferrite crystals structures (both with same fcc structure and lattice constant of 8.397 and $8.395 \AA,{ }^{20}$ respectively) and the fact that in our case the cobalt ferrite shell is quite substoichiometric in cobalt. Additionally, we performed the cation exchange of M21 in order to substitute the $\mathrm{Fe}^{2+}$ with $\mathrm{Mn}^{2+}$ cations, keeping the same concentration of the metal precursor ( $\mathrm{Mn}$ in this sample) used to synthesize the M21 Co sample. Like in the case of cation-exchange with $\mathrm{Co}^{2+}$ cations, the outcome of this reaction was again core/shell NPs, as displayed in Figure $1 \mathrm{j}-\mathrm{l}$ : M21 Mn sample shows monodispersed spherical NPs with mean diameter $\mathrm{d}=22 \pm 1.3 \mathrm{~nm}$ (Figure 1c). HRTEM imaging (Figure 1f) of M21_Mn indicates that the NPs are single crystals, and the effect of $\mathrm{Mn}$ introduction is visible in the STEM-EDS maps and related compositional profiles (Figure $1 \mathrm{j}-1$ ). Again, the corresponding $\mathrm{M}$-shaped profile indicates that manganese just gave rise to the formation of an external $\mathrm{Mn}_{x} \mathrm{Fe}_{3-x} \mathrm{O}_{4}$ shell, with $x$ equal to 0.25 \pm 0.08 and a mean shell thickness of $2.3 \pm 0.5 \mathrm{~nm}$.

In order to gain a deeper understanding of the $\mathrm{CE}$ reaction in iron oxide particles, we performed it between iron and cobalt in smaller iron oxide nanoparticles. Indeed, it is known that size is a crucial factor for iron oxide particles synthesized by a thermal decomposition approach, as the NPs varies from fully oxidized maghemite $\left(\gamma-\mathrm{Fe}_{2} \mathrm{O}_{3}\right)$ to a magnetite $\left(\mathrm{Fe}_{3} \mathrm{O}_{4}\right)$ / maghemite core/shell structure in the short span going from 8 to $12 \mathrm{~nm} .^{32}$ Then, by synthesizing NPs around $10 \mathrm{~nm}$ in size, we chose an intermediate condition where the oxidation still partially affects the magnetite NPs without giving rise to a proper maghemite shell (as expected for NPs bigger than 12 $\mathrm{nm}$ ) and without oxidizing the whole NPs to maghemite (as expected for NPs smaller than $8 \mathrm{~nm}) .^{33}$ The possible presence of maghemite plays a pivotal role with regards to CE reactions since its structure contains vacancies in the octahedral sites, contrary to magnetite. It has been reported that cation diffusion through interstitial sites is greatly improved by the presence of vacancies (internal or on the particles surface), dislocations, stacking faults, and possibly grain boundaries inside the NPs. ${ }^{13,17}$ Therefore, the presence of a partially oxidized, defective surface could enhance the cation exchange between $\mathrm{Fe}$ and $\mathrm{Co}$ or $\mathrm{Mn}$ at the surface of the magnetite NPs.

Thus, taking into account all the possible above-mentioned effects related to the use of smaller iron particles as starting material, we synthesized a sample, named M10, of monodispersed spherical $\mathrm{Fe}_{3} \mathrm{O}_{4} \mathrm{NPs}$ with mean size of $10.4 \pm 0.9$ $\mathrm{nm}$, following again the approach developed by Park et al. and described in the Experimental Section. TEM and HRTEM analysis conducted on M10 (Figure 2a,d) confirms its uniform shape and monodisperse size, while indicating that the monocrystalline structure is also maintained in smaller particles. We then used this sample as a starting material to carry out the $\mathrm{CE}$ protocol and substitute the $\mathrm{Fe}^{2+}$ with either $\mathrm{Co}^{2+}$ or $\mathrm{Mn}^{2+}$. This further choice was dictated by the interest in studying the effect of modifying the magnetic response of a given starting magnetic material by partially substituting its

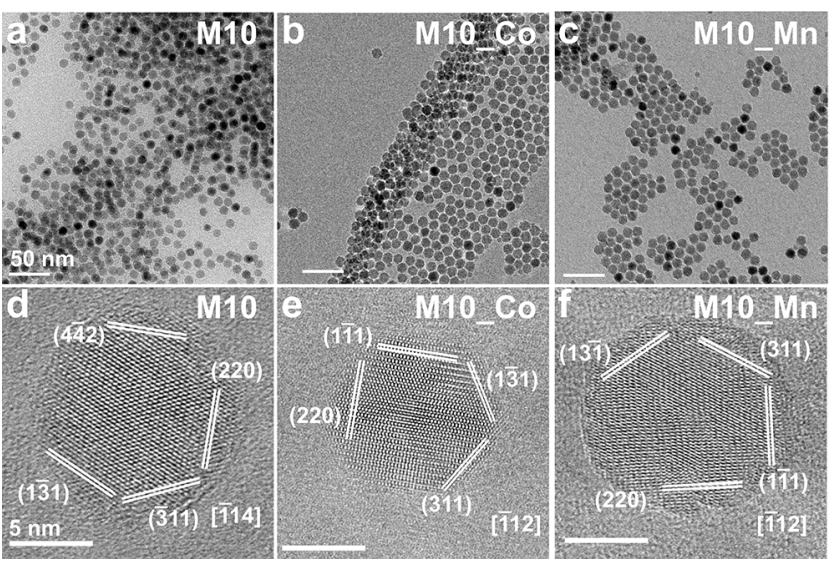

Figure 2. Conventional TEM and HRTEM images of samples M10 $(\mathrm{a}, \mathrm{d})$, M10_Co $(\mathrm{b}, \mathrm{e})$, and M10_Mn $(\mathrm{c}, \mathrm{f})$.

iron cations with magnetically harder or softer cations (Co or Mn, respectively).

We used the same Co and Mn precursor concentration in the two CE reactions in order to minimize the number of variables and provide a more straightforward comparison. Then, in performing the CE reactions, we chose the maximum concentration of the $\mathrm{Co}$ and $\mathrm{Mn}$ precursors that preserved both size and shape of the M10 NPs in the final products. TEM images of M10 Co and M10 Mn samples are reported in Figure 2b,c and show monodispersed spherical NPs with a mean diameter of $10.8 \pm 0.8$ and $10.9 \pm 0.8 \mathrm{~nm}$, respectively, retaining both shape and size of the M10 sample's NPs. Figure 2e,f shows HRTEM images of both M10_Co and M10_Mn samples, while $\mathrm{Fe}, \mathrm{Co}$ and $\mathrm{Mn}$ STEM-EDS mapping and related compositional profiles are displayed in Figure $3 \mathrm{a}-\mathrm{c}$ and
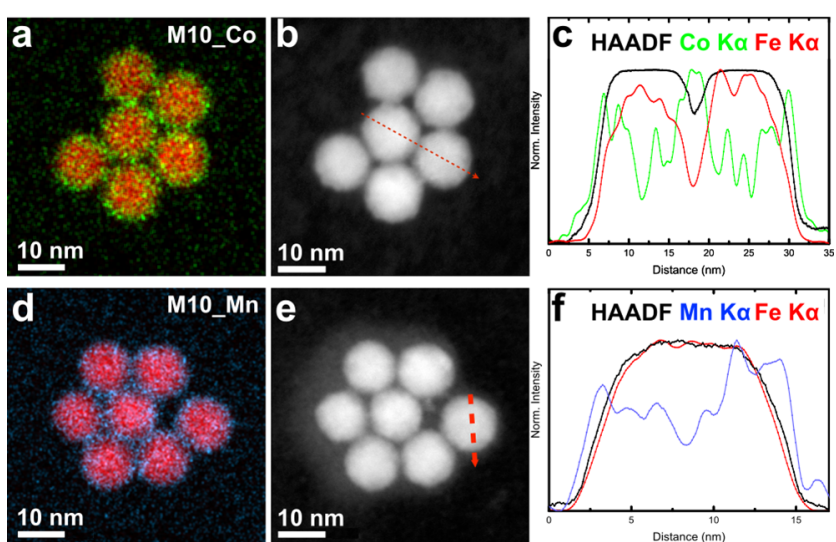

Figure 3. STEM-EDS maps (a,d), corresponding HAADF-STEM images (b,e) and compositional profiles (c,f) of samples M10_Co and M10_Mn. The same data are also reported as EDS single-element maps in Figure SI_3 for improved readability.

$\mathrm{d}-\mathrm{f}$. Again, analogously to what is found in the sample M21_Co, all the results point out to Co and Mn being mainly located in an external cobalt/manganese ferrite shell. The composition averaged over the whole NPs, was found to be $\mathrm{Co}_{x} \mathrm{Fe}_{3-x} \mathrm{O}_{4}$ or $\mathrm{Mn}_{y} \mathrm{Fe}_{3-y} \mathrm{O}_{4}(x=0.09 \pm 0.02$ and $y=0.11 \pm$ $0.02)$, and the corresponding mean shell thicknesses were equal to $2.2 \pm 0.2 \mathrm{~nm}$ and $2.1 \pm 0.4 \mathrm{~nm}$, respectively.

Moreover, we confirmed that increasing the concentration of $\mathrm{Mn}$ precursor in M10 NPs produces size dispersion and 
faceting in the final samples M10_Mn hc1 and M10_Mn hc2 (Figure SI_2b,c).

So far, it can be inferred that the intrinsic defectivity of the particle surface helps the cation exchange between iron and guest cations in the atomic layers closer to the external ones. These results are analogous to those shown in the paper of Luo et al., where similar reactions were performed resulting in the formation of $\mathrm{Fe}_{3} \mathrm{O}_{4} @ \mathrm{NiFe}_{x} \mathrm{O}_{y}$ core/shell NPs. ${ }^{34}$ This is also in accordance to the experimental evidence reported in our recent work, where we showed that the outcomes of the CE protocol vary depending on the availability of the doping species: for a low amount of $\mathrm{Mn}$ precursor, the $\mathrm{CE}$ gave rise to a thin shell of manganese ferrite substoichiometric in $\mathrm{Mn}$, while when the amount of Mn precursor was increased, the external layers of the pristine magnetite NPs were not able to further exchange iron cations, resulting in the overgrowth of a thick external $\mathrm{Mn}_{3} \mathrm{O}_{4}$ shell that modified the overall physical properties of the samples. ${ }^{26}$

In order to confirm the hypothesis of defectivity being the key parameter for a more effective cation exchange, we applied the $\mathrm{CE}$ protocol starting from a defective iron oxide and analyzed if this feature could give rise to an increased amount of exchanged cations.

Taking again into account that recently reported for chalcogenides compounds, ${ }^{13,17}$ we further synthesized a sample of iron oxide NPs, namely wüstite $\left(\mathrm{Fe}_{x} \mathrm{O}\right)$, which is a nonstoichiometric phase $\left(\mathrm{Fe}_{x} \mathrm{O}\right.$, with $\left.0.84<x<0.95\right)$ with fcc crystal structure, but containing a large number of iron vacancies. ${ }^{35-37}$ We followed the approach described by Hufschmind et al. ${ }^{38}$ trying to produce NPs with size very similar to the M10 samples, in order to study the effect of solely altering the crystal structure on the CE effectiveness. The sample we obtained was constituted by $\mathrm{Fe}_{x} \mathrm{O}$ NPs with a mean diameter $d=9.1 \pm 0.6 \mathrm{~nm}$ and was then named W9. After the synthesis, it was purified and stored in a glovebox under argon atmosphere in order to prevent the oxidation to magnetite. For this reason, the XRD analysis of the W9 sample was performed within the first $30 \mathrm{~min}$ from its exposure to air. Thus, the W9 XRD pattern, shown in Figure 4a, was indexed, and the (111), (200), (220), and (311) planes of the cubic wüstite structure (JCPDS card No. 84-0302) were identified. However, increasing the air exposure to 2 and $16 \mathrm{~h}$, the asprepared wüstite nanoparticles showed the distinct features of the magnetite structure with the presence of (311), (400), (511), and (440) peaks (JCPDS card No. 82-1533) due to the partial transformation of the $\mathrm{Fe}_{x} \mathrm{O}$ sample to magnetite via surface oxidation (Figure SI_4).

We then applied once more the cation exchange synthetic approach on the W9 sample under nonoxidizing conditions while carefully keeping the same concentration of the Co and Mn precursors used to synthesize the M10_Co and M10_Mn samples, respectively. In agreement with that reported in Table 1, we called the two further samples W9_Co and W9_Mn, respectively. Figure 4c,d shows the conventional TEM images of the latter samples, both of them being monodispersed spherical NPs with mean diameter $d=9.1 \pm 0.6 \mathrm{~nm}$ that retained shape and size of the starting material. The particles' shell constituting both samples took a stable ferrite crystal structure after the CE reaction, as shown by HRTEM imaging displayed in Figure 4f,g. It is worth noting that the structural rearranging associated with the $\mathrm{CE}$ still permitted discriminating the presence of the unperturbed wüstite cores by performing the inverse FFT analysis of the diffraction peaks
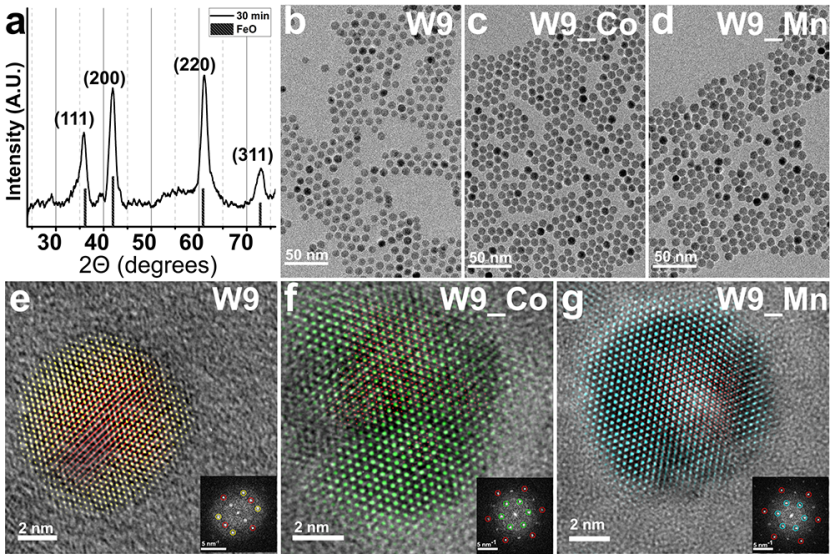

Figure 4. XRD pattern of sample W9 (a); conventional TEM and HRTEM images of samples W9 (b,e), W9_Co (c,f), and W9_Mn $(\mathrm{d}, \mathrm{g})$. HRTEM images of panels e-g are color-coded by inverse FFT to highlight the presence of wüstite cores, with the respective 2D-FFT patterns shown as insets: the diffraction spots are color-coded to identify those coming from the wüstite cores (in red) and from the ferrite shells (in yellow, green, and cyan, respectively). $\{311\}$ spots of magnetite (JCPDS card no. 82-1533) and a combination of $\{111\}$ and $\{220\}$ spots of wüstite (JCPDS card no. 84-0302) were used on the IFFT of W9, $\{220\}$ spots of magnetite and wüstite were used elsewhere.

associated with the phases constituting the core and the shell, respectively.

EDS-STEM chemical maps and corresponding elemental profiles, reported in Figure 5a-c (sample W9_Co), and Figure
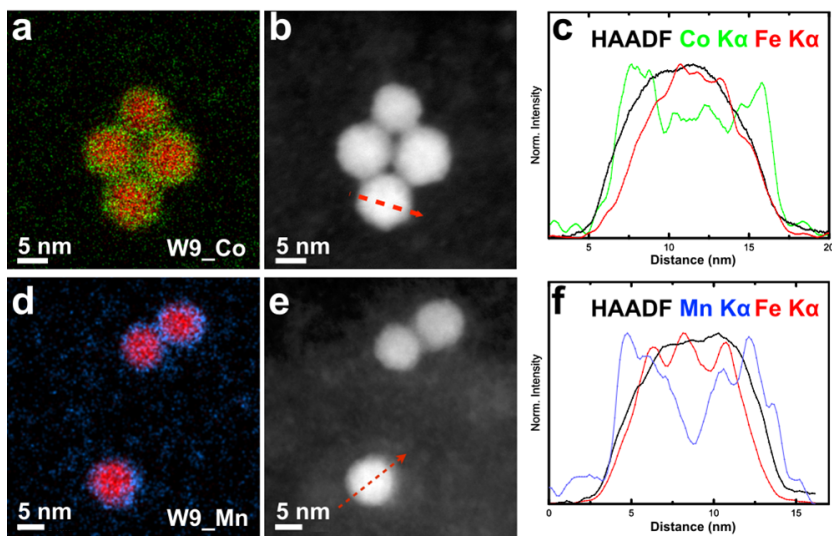

Figure 5. STEM-EDS maps (a,d), corresponding HAADF-STEM images (b,e) and compositional profiles (c,f) of samples W9_Co and W9_Mn. The same data are also reported as EDS single-element maps in Figure SI_5 for improved readability.

5d-f (sample W9_Mn), indicate that the NPs still kept the profiles of core/shell structures. Nevertheless, dramatic differences in the $\mathrm{CE}$ effectiveness are immediately apparent. In the case of pristine wüstite NPs, $\mathrm{CE}$ gave rise to much thicker ferrite shells, whose thicknesses are equal to $3.1 \pm 0.5$ $\mathrm{nm}$ for W9_Co and $2.5 \pm 0.7 \mathrm{~nm}$ for W9_Mn, respectively. This result was associated with a higher iron-to-guest cations exchange in the samples originated from the wüstite particles than what previously observed for magnetite, as evidenced by the composition of the post-CE NPs: when measured on the whole nanoparticles, $\mathrm{x}$ and $\mathrm{y}$ values of the $\mathrm{Co}_{x} \mathrm{Fe}_{3-x} \mathrm{O}_{4}$ and $\mathrm{Mn}_{y} \mathrm{Fe}_{3-y} \mathrm{O}_{4}$ shell were equal to $0.73 \pm 0.05$ and $0.61 \pm 0.05$, 
respectively. Then, changing the structure of the starting material from magnetite to the intrinsically defective wüstite boosted the efficiency of the $\mathrm{CE}$ and further pushed the $\mathrm{CE}$ front toward the inner part of the NPS, thus giving rise to thicker mixed ferrite shells and smaller unperturbed cores. The formation of a core/shell system corresponds to a chemically steady state, where the formation of a mixed ferrite shell of increasing thickness progressively reduces the number of vacancies available to push the $\mathrm{CE}$ exchange front toward the center of the NPs. Once in the most external layers of NPs, the $\mathrm{CE}$ has given rise to the formation of a stoichiometric mixed ferrite, it does not allow the further exchange of cations, and the CE front is stopped. This kind of mechanism was clearly described in ref 39 by computational simulations of CE in chalcogenides nanocrystals, which showed it could happen as a layer-by-layer reaction. This implies that it is a self-limiting reaction because the exchange rate fast decreases while the exchange front moves toward the center of the particles, until it becomes null, thus leaving the center of the particles unaffected by CE. Hence, despite the small size of the NPs, the products of these reactions were still core/shell NPs, in opposition to the results reported in the few works devoted to CE performed on iron oxide particles.

Further considerations can be done on what is described in the work of Sytnyk et al. concerning the $\mathrm{CE}$ on $\mathrm{Fe}_{x} \mathrm{O}-\mathrm{CoFe}_{2} \mathrm{O}_{4}$ core/shell heterostructures. ${ }^{18}$ This system had been already presented and studied in a paper of Bodnarchuk et al., ${ }^{40}$ but some aspects of the discussion on the evolution of that core/ shell system with CE appear quite debatable. First, the authors affirmed that in the initial core/shell system, prior to any CE reaction, the volume occupied by the wüstite core was $41 \%$ of the entire NPs and, considering that the diameter of the whole core/shells was $20 \mathrm{~nm}$, the diameter of the internal wüstite core could be calculated equal to about $15 \mathrm{~nm}$. Taking into account the small difference in density between cobalt ferrite and wüstite, the mean expected percentage of Co over the entire heterostructures' volume should be equal to $26 \%$, which is a quite higher value than that reported in the paper $(18 \%)$ of Sytnyk et al. Second, at RT, the starting core/shell structures did not display any kind of exchange coupling between the antiferromagnetic (AFM) wüstite core and the ferrimagnetic (FiM) cobalt ferrite shell, since the core is in a paramagnetic state (the Neél temperature of wüstite is $200 \mathrm{~K}$ ), which hinders any coupling with the FiM cobalt ferrite shell. Thus, the authors report that a $\mathrm{CE}$ reaction between $\mathrm{Fe}^{2+}$ and $\mathrm{Co}^{2+}$ was performed to transform the $\mathrm{Fe}_{x} \mathrm{O}$ core into a $\mathrm{Fe}_{x-y} \mathrm{Co}_{y} \mathrm{O}$ in order to increase the Neél temperature of the AFM core up to value close to RT. However, neither a full and solid explanation nor reasonable models were provided to illustrate how the $\mathrm{Co}^{2+}$ could diffuse via a stoichiometric cobalt ferrite to the wüstite core and partially substitute its $\mathrm{Fe}^{2+}$ cations. In fact, a stoichiometric cobalt ferrite should be a vacancy-free structure, which would make it very hardly prone to allow such a CE reaction, as discussed above. However, these significant discrepancies could be explained supposing that the shell considered by the authors as constituted by a stoichiometric cobalt ferrite was in fact either a defective cobalt ferrite substoichiometric in cobalt (i.e., $\mathrm{Co}_{x} \mathrm{Fe}_{2} \mathrm{O}_{4}, x<1$ ) or a partially cobalt-doped magnetite (i.e., $\mathrm{Co}_{x} \mathrm{Fe}_{3-x} \mathrm{O}_{4}, x<1$ ), being the crystal structure and the relevant lattice parameter of both these compounds compatible with what observed by XRD by the authors, with the small change in the lattice parameter of the wüstite core ascribed to its doping with some Co cations.
In any case, the fact that the shell could be actually constituted by such a kind of defective structures could in turn make it capable to provide some vacancies that act as channel to allow the CE occurrence described in the paper of Sytnyk et al., confirming that the diffusion of the cobalt ions through the cobalt ferrite shell was likely due to the presence of vacancies in its structure. In addition, the authors reported that the volume of the cobalt-modified AFM core was reduced to just the $8 \%$ of the total after the CE reaction, whereas the rest of the heterostructure was again constituted by FiM cobalt ferrite. Then, taking again into account the slight difference in density between cobalt ferrite and wüstite, the calculated mean content of cobalt over the entire heterostructures should be higher than $32 \%$, since we are not taking into account the amount of cobalt contained in the Co-doped wüstite core, which is not reported and must be ruled out, thus lowering the Co actual content. Interestingly, this minimal value is still higher than what reported by Sytnyk and coauthors (27\%), again indicating that the cobalt content expected for the formation of a stoichiometric $\mathrm{CoFe}_{2} \mathrm{O}_{4}$ shell was not reached even after the CE. ${ }^{18}$ Furthermore, the authors explained the growth of the cobalt ferrite shell as a partial oxidation of the $\mathrm{Fe}^{2+}$ present in the wüstite core due to oxygen diffusion through the shell to the core. However, in the work of Bodnarchuk et al., ${ }^{40}$ the same starting core/shell heterostructures are reported to be stable against the wüstite core oxidation over time, despite the lower than expected content of cobalt for a stoichiometric ferrite shell. This in turn suggests that the CE process is not just facilitating the iron partial oxidation, as stated by Sytnyk and coauthors, ${ }^{18}$ but it is likely changing the heterostructures in a more complex way: first, $\mathrm{Co}^{2+}$ ions move toward the wüstite core exploiting the cationic vacancies contained in the ferrite shell, thus giving rise to its growth at the expense of the core; second, it is promoting the production of anionic vacancies that are able to reach the wüstite core through the cobalt ferrite shell leading to its partial oxidation.

Finally, we performed an in-depth magnetic behavior characterization to confirm the core/shell intrinsic nature of our systems by studying the effect of the CE on the magnetitebased and wüstite-based NPs, whose diameters are 10 and 9 $\mathrm{nm}$, respectively.

In all the $10 \mathrm{~nm}$ sized magnetite-based samples, all the $T_{\mathrm{B}}$ and $T_{\text {IRR }}$ values of the zero field cooled and field cooled (ZFCFC) curves fall within the exploited temperature range, meaning that the transition from a magnetically blocked to an unblocked state takes place below $300 \mathrm{~K}$ for all the samples. The variation of the maximum of the $\mathrm{ZFC}$ curves (corresponding to the temperature $T_{\mathrm{B}}$ ) and of the point where ZFC and FC curves superimpose $\left(T_{\text {IRR }}\right)$ from M10 to M10 Mn and M10 Co confirms the results obtained by TEM, with steady $\mathrm{T}_{\mathrm{B}}$ values for M10 and of M10_Mn, accompanied by a slight shift toward higher temperatures for M10_Co and a stable $\Delta T\left(\Delta T=T_{\text {IRR }}-T_{\mathrm{B}}\right)$ value for all the samples (Table 2).

Table 2. Parameters from the ZFC-FC Curves of MagnetiteBased Samples

$\begin{array}{lccc}\text { sample } & T_{\mathrm{B}}(\mathrm{K}) & T_{\mathrm{IRR}}(\mathrm{K}) & \Delta T(\mathrm{~K}) \\ \text { M10 } & 104 & 150 & 46 \\ \text { M10_Co } & 143 & 190 & 47 \\ \text { M10_Mn } & 109 & 150 & 41\end{array}$


These data are in very good accordance with the results of size analysis obtained by TEM, which showed no variation in the mean size between M10, M10_Mn, and M10_Co while keeping the same standard deviation for all the samples. Then, these results first indicate that the $\mathrm{CE}$ reactions increase the effective anisotropy constant of only the M10_Co sample because its mean size and relevant distribution did not change, as well as that of the M10_Mn one. However, even for the latter sample, a slight $T_{\mathrm{B}}$ increase was observed. As the $\mathrm{Fe} \rightarrow$ $\mathrm{Mn}$ exchange is expected softening the overall magnetic character of the treated nanoparticles, such a $T_{\mathrm{B}}$ increase has to be ascribed to a partial increase of the NPs superficial ordering, which basically results as a sort of slight rise of the effective single magnetic domain volume, in accordance with what we reported in our recent work ${ }^{26}$ (Figure $6 a-c$ ).
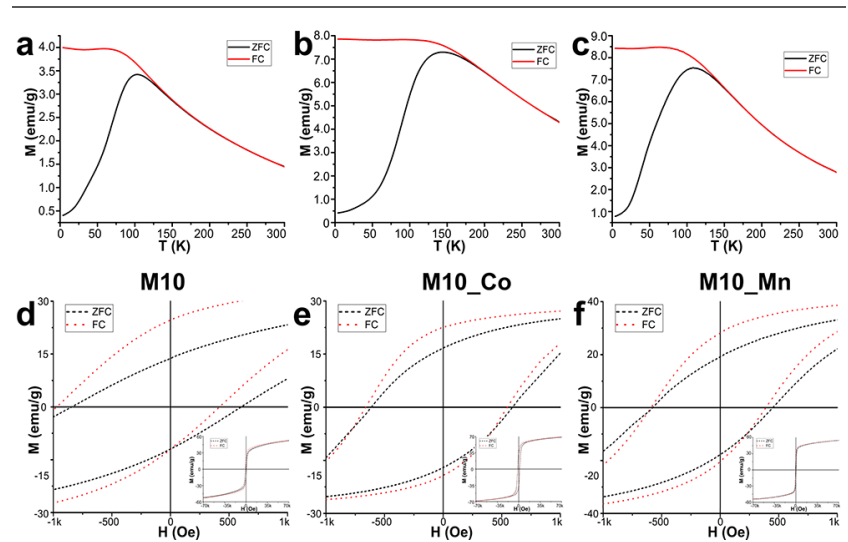

Figure 6. ZFC-FC magnetization curves of samples M10 (a), M10_Co (b), and M10_Mn (c). The corresponding parameters are reported in Table 2; zero field cooled (dashed lines) and field cooled (dotted lines) hysteresis loops of samples M10 (d), M10_Co (e), and M10_Mn (f) recorded at $4 \mathrm{~K}$ : the main panels represent the low field region, while the full corresponding hysteresis loops are shown in inset and as full-sized panels in Figure SI_6a-c. All the corresponding magnetic parameters are reported in Table 3.

Furthermore, it is noteworthy looking at the $\Delta T$ values measured for all the M10-based samples, which are basically kept constant. At a first glance it could be surprising that so high values could come from NPs homogeneous in size, shape and composition. However, this evidence could be ascribed to a possible spin-glass-like behavior of the samples occurring at low temperature, as also indicated by the plateau reached by the FC curves in that thermal range.

The magnetic response of the magnetite-based samples was further studied in the high magnetic field regime by recording hysteresis loops at $4 \mathrm{~K}$ and at $\mathrm{RT}$, corresponding to the magnetically blocked and unblocked states of the NPs (Figure $6 \mathrm{~d}-\mathrm{f}$, Figure SI_7a-c and Table SI_1). Also, additional fieldcooled (FC) hysteresis loops were collected to confirm the presence of shell and investigate the characteristics of the core/ shell interface, as explained more in-depth in the following. The magnetic response of the samples was analyzed through the typical hysteretic parameters, i.e., saturation magnetization $\left(M_{S}\right)$, mean remanence $\left(M_{R}=\left(\left|M_{R+}\right|+\left|M_{R-}\right|\right) / 2\right)$, where $M_{R+}$

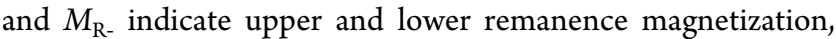
respectively), loop vertical shift $\left(\Delta M_{\mathrm{R}}=\left(\left|M_{\mathrm{R}+}\right|-\left|M_{\mathrm{R}-}\right|\right) / 2\right)$, mean coercivity $\left(H_{\mathrm{C}}=\left(\left|H_{\mathrm{C} 1}\right|+\left|H_{\mathrm{C} 2}\right|\right) / 2\right.$, where $H_{\mathrm{C} 1}$ and $H_{\mathrm{C} 2}$ are the negative and positive coercive fields, respectively) and exchange bias coercivity $\left(\mathrm{H}_{\mathrm{E}}=\left(\left|H_{\mathrm{C} 1}\right|-\left|H_{\mathrm{C} 2}\right|\right) / 2\right)$, the latter one providing a quantitative measurement of the horizontal shift observed in hysteresis loops.

As already observed from the ZFC-FC curves in the low field regime, the M10 and the M10_Mn samples show some common traits, while the introduction of cobalt in M10 Co again determines more radical variations. CE reactions boost both remanence and saturation magnetization: in the former the increase is already noticeable for M10_Mn and is apparent for M10_Co; in the latter the introduction of $\mathrm{Mn}$ gives rise to a slight variation from M10 $(60.1 \mathrm{emu} / \mathrm{g})$ to M10 Mn $(60.8$ $\mathrm{emu} / \mathrm{g})$, while the saturation magnetization of $\overline{\mathrm{M}} 10 \_\mathrm{Co}$ is boosted to $74 \mathrm{emu} / \mathrm{g}$ (Table 3). However, all the saturation values at $4 \mathrm{~K}$ are lower than the bulk values expected for pure phases $\left(M_{\mathrm{S}}=100 \mathrm{emu} / \mathrm{g}, 110 \mathrm{emu} / \mathrm{g}\right.$ and $90 \mathrm{emu} / \mathrm{g}$ for $\mathrm{Fe}_{3} \mathrm{O}_{4}$, $\mathrm{MnFe}_{2} \mathrm{O}_{4}$ and $\mathrm{CoFe}_{2} \mathrm{O}_{4}$, respectively). ${ }^{41-43}$ This is not surprising for small-sized NPs systems, where it indicates that structural disorder affects the NPs' surface of the magnetite-based sample and prevents the proper orientation of all the spins, ${ }^{21}$ but it is also expected in the case of core/ shell systems, whose magnetic features come from a combination of the parameters of the pure phases.

The fundamental role of surface effects on the magnetic response of magnetite-based samples is also highlighted by the coercivity. In the case of the pristine M10 sample, $H_{\mathrm{C}}$ at $4 \mathrm{~K}$ is consistently higher than the results obtained for $\mathrm{Fe}_{3} \mathrm{O}_{4} \mathrm{NPs}$ of

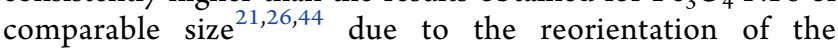
superficial unbalanced spins, which translates into a magnetic hardening. Conversely, the presence of $\mathrm{Mn}$ and $\mathrm{Co}$ as mixed shells in M10_Mn and M10_Co modifies the initial coercivity of M10 in opposite ways, depending on the magnetic characteristics of each shell: the introduction of $\mathrm{Mn}$ has a magnetic softening effect with respect to magnetite, while Co induces a hardening, as evidenced by the gradual increase in anisotropy constant values going from $\mathrm{MnFe}_{2} \mathrm{O}_{4}$ to $\mathrm{Fe}_{3} \mathrm{O}_{4}$ and $\mathrm{CoFe}_{2} \mathrm{O}_{4}$ (bulk values being $2.5 \times 10^{3} \mathrm{~J} / \mathrm{m}^{3}, 1.9 \times 10^{4} \mathrm{~J} / \mathrm{m}^{3}$

Table 3. Parameters of ZFC and FC Hysteresis Loops Recorded at $4 \mathrm{~K}$ for Magnetite-Based Samples

\begin{tabular}{|c|c|c|c|c|c|c|c|c|}
\hline \multirow[b]{2}{*}{ sample } & \multicolumn{8}{|c|}{ ZFC hysteresis } \\
\hline & $H_{\mathrm{Cl}} @ 4 \mathrm{~K}(\mathrm{Oe})$ & $H_{\mathrm{C} 2} @ 4 \mathrm{~K}(\mathrm{Oe})$ & $H_{\mathrm{C}} @ 4 \mathrm{~K}(\mathrm{Oe})$ & $H_{\mathrm{E}}^{\mathrm{SEB}}(\mathrm{Oe})$ & $M_{\mathrm{S}} @ 4 \mathrm{~K}(\mathrm{emu} / \mathrm{g})$ & $M_{\mathrm{R}} @ 4 \mathrm{~K}(\mathrm{emu} / \mathrm{g})$ & $\Delta M_{\mathrm{R}}(\mathrm{emu} / \mathrm{g})$ & $M_{\mathrm{R}} / M_{\mathrm{S}}$ \\
\hline M10 & -842 & 605 & 724 & -119 & 60.1 & 12.8 & 0.9 & 0.21 \\
\hline M10_Co & -1840 & 1780 & 1810 & -30 & 74.5 & 33.7 & 0.4 & 0.45 \\
\hline \multirow[t]{3}{*}{ M10_Mn } & -556 & 449 & 503 & -54 & 60.8 & 18.4 & 0.7 & 0.30 \\
\hline & \multicolumn{8}{|c|}{ FC hysteresis } \\
\hline & $H_{\mathrm{C} 1} @ 4 \mathrm{~K}(\mathrm{Oe})$ & $H_{\mathrm{C} 2} @ 4 \mathrm{~K}(\mathrm{Oe})$ & $H_{\mathrm{C}} @ 4 \mathrm{~K}(\mathrm{Oe})$ & $H_{\mathrm{E}}^{\mathrm{CEB}}(\mathrm{Oe})$ & $M_{\mathrm{S}} @ 4 \mathrm{~K}(\mathrm{emu} / \mathrm{g})$ & $M_{\mathrm{R}} @ 4 \mathrm{~K}(\mathrm{emu} / \mathrm{g})$ & $\Delta M_{\mathrm{R}}(\mathrm{emu} / \mathrm{g})$ & $M_{\mathrm{R}} / M_{\mathrm{S}}$ \\
\hline M10 & -971 & 420 & 695 & -276 & 60.0 & 18.4 & 6.3 & 0.31 \\
\hline M10_Co & -1988 & 1645 & 1817 & -172 & 77.7 & 41.9 & 3.3 & 0.54 \\
\hline M10_Mn & -589 & 390 & 490 & -99 & 60.1 & 24.3 & 3.9 & 0.40 \\
\hline
\end{tabular}


and $3.0 \times 10^{5} \mathrm{~J} / \mathrm{m}^{3}$, respectively). ${ }^{22,45,46}$ These general trends are respected in the present case and the $H_{\mathrm{C}}$ values of M10_Mn and M10_Co are, respectively, lower and higher than that of M10.

An indication on the role played by the superficial magnetic disorder is given by the presence of exchange bias (EB) effects, due to the magnetic coupling of NP's core and shell layer. ${ }^{20-24}$ EB determines a horizontal shift of the hysteresis loops that manifests as a change of both negative and positive measured coercive field, $\left|H_{\mathrm{C} 1}\right|$ and $\left|H_{\mathrm{C} 2}\right|$, respectively. Moreover, EB can be divided in two diverse categories: spontaneous exchange bias (SEB), affecting the coercivity of the ZFC hysteresis loops, and conventional exchange bias (CEB), which modifies the coercivity values on hysteresis loops of samples cooled under a strong magnetic field (FC hysteresis loops). The systematic drop of $H_{\mathrm{E}}{ }^{\mathrm{SEB}}$ after the introduction of $\mathrm{Mn}$ and Co further confirms this interpretation: the formation of a thin mixed $\mathrm{Fe} /$ $\mathrm{M}(M=\mathrm{Mn}, \mathrm{Co})$ shell has a curing effect that decreases the superficial disorder of the NPs, leading to a drop in $H_{\mathrm{E}}^{\mathrm{SEB}}$ and $H_{\mathrm{E}}^{\mathrm{CEB}}$ for M10_Mn and M10_Co as detected in the ZFC and FC hysteresis loops, respectively (Table 3 ).

Then, CE reactions promote a disordered-to-ordered interface change of M10, which in turn determines a marginal growth of the core magnetic domains from M10 to M10_Mn and M10_Co, as shown by the increase of their $T_{B}$ values, and the formation of a proper shell with a more ordered core/shell interface that cuts down the disorder-driven asymmetry effects. The changes observed for $H_{\mathrm{E}}{ }^{\mathrm{SEB}}$ and the $H_{\mathrm{E}}{ }^{\mathrm{CEB}}$ on the CEtreated samples are then in full agreement with what we already reported in our recent paper. $^{26}$

A further consideration is deserved by the vertical shift observed in the ZFC and FC hysteresis loops for all the M10based samples (indicated as $\Delta M_{\mathrm{R}}$ in Table 3). As reported in ref 47 , this shift could be further ascribed to a spin-glass-like magnetic behavior of the NPs, whose main contribution is given by the superficial disordered spins. CE partially cures this disorder, ${ }^{26}$ as further indicated by loops'vertical shift, which is lowered but not erased when passing from M10 to M10_Co and M10_Mn samples, thus indicating their maintained spinglass-like $\bar{b}$ ehavior.

The comparison between pristine and CE samples in the case of wüstite-based samples is not as straightforward as for the magnetite-based ones and the effects of CE reactions are different. The pristine wüstite W9 and its offshoots W9 Mn and W9_Co are synthesized and maintained in oxygen-free conditions during the $\mathrm{CE}$ reactions that lead to the formation of $\mathrm{M}_{x} \mathrm{Fe}_{3-x} \mathrm{O}_{4}$ shells $(\mathrm{M}=\mathrm{Co}, \mathrm{Mn})$ in W9_Mn and W9_Co. Then, the superficial oxidation that gives rise to the core/shell in W9 is an inevitable side effect of its later exposition to air but does not represent the initial condition of W9 NPs before the CE reactions with $\mathrm{Mn}$ and $\mathrm{Co}$. A CE reaction was repeated on oxidized wüstite W9 NPs to prove the effect of superficial oxidation of the starting NPs on the final product of synthesis. The resulting sample (W9ox Mn, see Figure SI 8) did not maintain the shape, size, and $\overline{M n}$ content previously observed in W9 Mn, but gave rise to flowerlike core/shell NPs with iron oxide cores and manganese oxide shells. This result, which closely recalls our recent study on $\mathrm{Fe}_{3} \mathrm{O}_{4} / \mathrm{Mn}_{3} \mathrm{O}_{4}$ core/shell systems, ${ }^{26}$ further proves that the presence of nonoxidized wüstite NPs is a mandatory condition to successfully conduct these $\mathrm{CE}$ reactions, since the presence of a magnetite shell shields the NPs and inhibits the CE.
The thermally dependent magnetic response of the wüstitebased samples was at first investigated by ZFC-FC measurements in a low magnetic field regime. The blocking and irreversibility temperatures $T_{\mathrm{B}}$ and $T_{\mathrm{IRR}}$ are within the exploited thermal range and fall below $\mathrm{RT}$, with reduced $T_{\mathrm{B}}$ values for the W9_Mn and W9_Co samples (Figure 7a-c, Table 4).

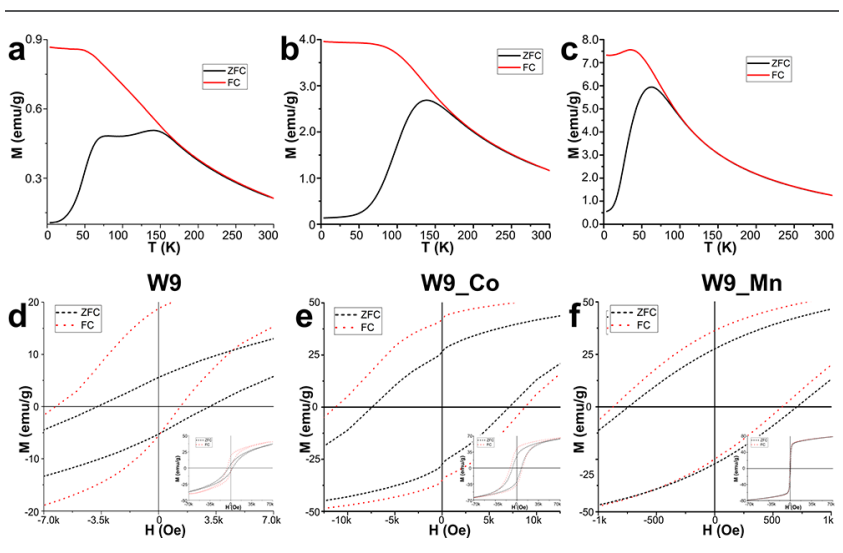

Figure 7. ZFC-FC magnetization curves of samples W9 (a), W9_Co (b) and W9_Mn (c). The corresponding parameters are reported in Table 4; zero field cooled (dashed lines) and field cooled (dotted lines) hysteresis loops of samples W9 (d), W9 Co (e), and W9 Mn (f) recorded at $4 \mathrm{~K}$ : the main panels represent the low field region, while the full corresponding hysteresis loops are shown in inset and as full-sized panels in Figure SI_6d-f. All the corresponding magnetic parameters are reported in Table 5.

Table 4. Parameters from the ZFC-FC Curves of WüstiteBased Samples

\begin{tabular}{lccc} 
sample & $T_{\mathrm{B}}(\mathrm{K})$ & $T_{\text {IRR }}(\mathrm{K})$ & $\Delta T(\mathrm{~K})$ \\
W9 & 80 (shoulder peak); 165 & 176 & 11 \\
W9_Co & 139 & 190 & 51 \\
W9_Mn & 77 & 114 & 37 \\
\hline
\end{tabular}

According to the size analysis, since the CE reactions did not cause any noticeable variation in shape, mean size and distribution, the reduced $T_{\mathrm{B}}$ should be attributed to variations in the effective magnetocrystalline anisotropy, while an increase in $\Delta T$ could indicate that the effect of the $\mathrm{CE}$ reaction is not perfectly uniform and creates mixed shells with a slight distribution in thickness, as expected looking at the concentration profiles of both $\mathrm{Co}$ and $\mathrm{Mn}$ in the respective sample. Moreover, the magnetization in the ZFC-FC curves of W9_Mn and W9_Co are 4 times higher than that observed in W9: given the antiferromagnetic nature of the core and the combined effect of small size and thick cation-exchanged shell, after the CE reaction, the magnetization is mostly commanded by the shell while contributions coming from the wüstite core are mostly covered. However, as already observed for the M10based samples, the $\Delta T$ values measured for all the W9-based samples show too high values, not expected from NPs quite homogeneous in size, shape, and composition. Again, this phenomenon could be also ascribed to a possible spin-glasslike behavior of the samples occurring at low temperature, and even in the case of these samples this hypothesis is corroborated by the appearance of the plateau in all the FC curves at low thermal range. 
Table 5. Parameters of ZFC and FC Hysteresis Loops Recorded at 4 K for Wüstite-Based Samples

\begin{tabular}{|c|c|c|c|c|c|c|c|c|}
\hline \multirow[b]{2}{*}{ sample } & \multicolumn{8}{|c|}{ ZFC hysteresis } \\
\hline & $H_{\mathrm{Cl}} @ 4 \mathrm{~K}(\mathrm{Oe})$ & $H_{\mathrm{C} 2} @ 4 \mathrm{~K}(\mathrm{Oe})$ & $H_{\mathrm{C}} @ 4 \mathrm{~K}(\mathrm{Oe})$ & ${H_{\mathrm{E}}}^{\mathrm{SEB}}(\mathrm{Oe})$ & $M_{\mathrm{S}} @ 4 \mathrm{~K}(\mathrm{emu} / \mathrm{g})$ & $M_{\mathrm{R}} @ 4 \mathrm{~K}(\mathrm{emu} / \mathrm{g})$ & $\Delta M_{\mathrm{R}}(\mathrm{emu} / \mathrm{g})$ & $M_{\mathrm{R}} / M_{\mathrm{S}}$ \\
\hline W9 & -3733 & 3109 & 3421 & -312 & 51.3 & 5.4 & 0.2 & 0.11 \\
\hline W9_Co & -7409 & 7088 & 7248 & -161 & 75.0 & 27.0 & 0.4 & 0.36 \\
\hline \multirow[t]{3}{*}{ W9_Mn } & -770 & 699 & 735 & -35 & 87.2 & 28.0 & 0.5 & 0.32 \\
\hline & \multicolumn{8}{|c|}{ FC hysteresis } \\
\hline & $H_{\mathrm{Cl}} @ 4 \mathrm{~K}(\mathrm{Oe})$ & $H_{\mathrm{C} 2} @ 4 \mathrm{~K}(\mathrm{Oe})$ & $H_{\mathrm{C}} @ 4 \mathrm{~K}(\mathrm{Oe})$ & $H_{\mathrm{E}}^{\mathrm{CEB}}(\mathrm{Oe})$ & $M_{\mathrm{s}} @ 4 \mathrm{~K}(\mathrm{emu} / \mathrm{g})$ & $M_{\mathrm{R}} @ 4 \mathrm{~K}(\mathrm{emu} / \mathrm{g})$ & $\Delta M_{\mathrm{R}}(\mathrm{emu} / \mathrm{g})$ & $M_{\mathrm{R}} / M_{\mathrm{S}}$ \\
\hline W9 & -6509 & 1317 & 3913 & -2596 & 48.0 & 12.2 & 6.6 & 0.25 \\
\hline W9_Co & -11309 & 8761 & 10035 & -1274 & 75.4 & 38.6 & 3.5 & 0.51 \\
\hline W9_Mn & -893 & 597 & 745 & -148 & 84.0 & 21.0 & 3.4 & 0.25 \\
\hline
\end{tabular}

On the other hand, the ZFC-FC curves of W9 has some interesting features that must be pointed out (Figure $7 \mathrm{a}$ ): first, the value of measured magnetization over the entire thermal range exploited are always very low, in agreement with that expected for NPs still partially constituted by a AFM compound. Moreover, two peaks can be identified in the ZFC curve at 80 and $165 \mathrm{~K}$, respectively, and their presence can be effectively put in relation with the NPs composition. The first peak at $80 \mathrm{~K}$ can be attributed to the unblocking of NPs that already underwent heavy oxidation to $\mathrm{Fe}_{3} \mathrm{O}_{4}$, as also suggested by the comparison with the blocking temperature measured for M10 magnetite NPs $\left(T_{B}=104 \mathrm{~K}\right.$ for M10), because the two $T_{B}$ values are proportionally scaled with the variation in volume observed between W9 and M10. A further confirmation was provided by the ZFC curve of a fully oxidized W9 sample, which features only one peak at $80 \mathrm{~K}$ (Figure SI 9).

On the other hand, the second peak at $165 \mathrm{~K}$, along with the higher magnetization observed up to RT, is consistent with the antiferromagnetic to paramagnetic (AFM-to-PM) transition of the antiferromagnetic (AFM) wüstite core and can be attributed to the combination of two separate effects: first, the profile of the ZFC and FC curves from $165 \mathrm{~K}$ to RT and the small temperature range between the peak and the superposition of the ZFC and FC curves $\left(T_{B}=165 \mathrm{~K}\right.$ vs $T_{\text {IRR }}=176 \mathrm{~K}$ ) suggest that the AFM-to-PM transition of the AFM cores takes place at a lower temperature than the Neél one of bulk wüstite $\left(T_{\mathrm{N}} \approx 200 \mathrm{~K}\right)$ due to size-related effects already observed for small-sized AFM oxides. ${ }^{48-50}$ Moreover, the presence of a high temperature peak is consistent with the unblocking of the $\mathrm{Fe}_{3} \mathrm{O}_{4}$ shell of the partially oxidized wüstite NPs and is consistent with previous results on partially oxidized nanosized wüstite systems of similar size, ${ }^{51-53}$ where the presence of high-temperature $T_{\mathrm{B}}$ was commanded by the formation of a ferrimagnetic shell. ${ }^{51}$ Overall, the low magnetization values observed in the ZFC-FC curves and the absence of Verwey transition $\left(T_{\mathrm{V}} \approx 120 \mathrm{~K}\right)$ indicate that the fast oxidation process already observed by XRD analysis was at its very early stages during the SQUID analysis.

The composite nature and mixed magnetic behavior of wüstite-based W9_Co and W9_Mn samples implies that the final magnetization and coercivity values come from the combination and interaction of the AFM core with the thin FiM shell. Indeed, given the AFM nature of the wüstite core, the FiM shell provides the main contribution to $M_{S}$ and $M_{R}$ at low temperature, while the core/shell interactions play a key role in defining the magnetic hardness, as expressed by $\mathrm{H}_{\mathrm{C}}$ and $\mathrm{H}_{\mathrm{E}}$. The effect of wüstite on the magnetic response of the W9_Co and W9_Mn samples is already evident from the shape of the hysteresis loops at $4 \mathrm{~K}$, which enclose bigger areas and close at higher fields compared to their magnetite-base counterparts (Figure $7 \mathrm{~d}-\mathrm{f}$, Table 5 ). Once again a core/shelllike magnetic response can be observed in all the samples: in W9 it is due to the superficial oxidation of wüstite to magnetite, while in W9_Mn and W9_Co CE reactions lead to proper core/shell systems.

In the case of W9, the magnetite shell formed due to the superficial oxidation of wüstite increases the saturation magnetization to $51 \mathrm{emu} / \mathrm{g}$, which is in good accordance with the result obtained for similar-sized, oxidized wüstite NPs. ${ }^{52,54,55}$ This saturation value is compatible with the presence of a wüstite/magnetite core/shell system, giving rise to intermediate values between those expected for pure wüstite and pure magnetite NPs (20 and $100 \mathrm{emu} / \mathrm{g}$, respectively). In fact, the presence of a thin, disordered oxidized shell can be easily pointed out by magnetic characterization because the formation of a minor FiM phase on a major AFM phase gives rise to a noticeable variation in the net magnetization of the overall system which would be less apparent by standard structural techniques like XRD due to the small size and high level of disorder of these newly formed very thin FiM layer. The thicker shells formed by CE reactions with Co and $\mathrm{Mn}$ command as well the magnetization of W9_Mn and W9_Co and give rise to heightened remanence and saturation values. These results are comparable with those of mixed magnetitebased phases and not compatible with $\mathrm{Fe}_{x} \mathrm{O} / \mathrm{M}_{y} \mathrm{O}_{z}(\mathrm{M}=\mathrm{Co}$, $\mathrm{Mn}$ ) core/shell systems solely composed by AFM phase; thus, the enhancement of $\mathrm{M}_{\mathrm{S}}$ comes from the partial oxidation of the wüstite NPs taking place during CE reactions, more pronounced in the external part of the starting $\mathrm{Fe}_{x} \mathrm{O}$ NPs, then leading to mixed FiM $\mathrm{M}_{x} \mathrm{Fe}_{3-x} \mathrm{O}_{4}$ shells $(\mathrm{M}=\mathrm{Co}, \mathrm{Mn})$. In fact, while the $\mathrm{M}_{\mathrm{S}}$ values of bulk $\mathrm{MnFe}_{2} \mathrm{O}_{4}$ and $\mathrm{CoFe}_{2} \mathrm{O}_{4}$ are lower than $\mathrm{Fe}_{3} \mathrm{O}_{4}$, the formation of a thicker shell means a shrinking of the wüstite core and, consequently, magnetization values that tend more toward those typical of the ferrite shell phases (Table 5).

The presence of a shell in the wüstite-based samples also plays a major role in determining their magnetic hardness, starting from the W9 sample, whose high $H_{\mathrm{C}}$ value at $4 \mathrm{~K}$ clearly confirms the presence of a nonoxidized wüstite core: even taking into account that tiny variations of size and/or composition can cause non-negligible differences in the features of such small-sized NPs, its mean coercivity $\left(H_{\mathrm{C}}=\right.$ $3.4 \mathrm{kOe}$ ) is higher than those of pristine and completely oxidized wüstite ${ }^{54,56}$ but is comparable with those of partially oxidized NPs. $^{52,57}$ The final evidence of the presence of a core/ shell system in W9 in given by SEB and CEB effects, which can be observed in the hysteresis loops recorded at $4 \mathrm{~K}$ upon zero 
field cooling and field cooling ( $T=70 \mathrm{kOe})$ (Table 5). In particular, the strong $H_{\mathrm{C}}{ }^{\mathrm{SEB}}$ value at $4 \mathrm{~K}$ and its absence at $\mathrm{RT}$ hold a particular interest because they prove the presence of a disordered AFM/FiM interface. The effect of its EB can be measured clearly at low temperature with the shell in a magnetically blocked state and becomes null at high temperature, when the shell is unblocked, as already shown by the ZFC-FC curves (Figure $7 a-c$ ). ${ }^{52,57}$ In fact, for W9 the EB effects are null at RT (Figure SI_7d-f and Table SI_2) but amount to huge SEB and CEB values in the ZFC and FC hysteresis $\left(H_{\mathrm{E}}^{\mathrm{SEB}}=0.3 \mathrm{kOe}, H_{\mathrm{E}}^{\mathrm{CEB}}=2.6 \mathrm{kOe}\right)$ at $4 \mathrm{~K}$ : this experimental evidence implies the strong presence of uncompensated spins and suggests the highly disordered nature of W9.

As already observed in the case of W9, the FiM shell is the main responsible for the magnetization at $4 \mathrm{~K}$ of W9_Mn and W9 $\mathrm{Co}$, enhancing once again the saturation magnetization and leading to high $M_{S}$ values for both samples (Table 5). The formation of well-defined AFM/FiM core/shell systems also explains the EB phenomena and the modification of magnetic hardness observed in W9 $\mathrm{Mn}$ and W9 Co: wüstite is magnetically harder than magnetite, thus the AFM cores enhance the coercivity of W9 $\mathrm{Mn}$ and W9 Co in comparison to M10_Mn and M10_Co, while the presence of thicker shells implies that their contribution to the overall magnetic response becomes more pronounced and dependent on each phase's magnetic hardness. This determines an overall modulated effect of softening for W9 $\mathrm{Mn}$ and hardening for W9 Co due to the formation of a $\overline{M n}_{x} \mathrm{Fe}_{3-x} \mathrm{O}_{4}$ and $\mathrm{Co}_{x} \mathrm{Fe}_{3-x} \overline{\mathrm{O}}_{4}$ shell, respectively. Then, the coercivity of W9_Mn can be interpreted as a strong shell-driven softening of W9, while the combination of a magnetically hard core and a thicker, hard shell makes W9_Co much harder than all the other samples. The SEB and CEBB effects in the ZFC and FC hysteresis loops were studied to point out the features of the core/shell interfaces of W9 Mn and W9_Co (Figure 7d-f, Table 5). $H_{\mathrm{E}}^{\mathrm{SEB}}$ highlights the nature of the core/shell interface formed as a result of the CE reactions: ${H_{\mathrm{E}}}^{\mathrm{SEB}}$ at $4 \mathrm{~K}$ is non-null for both samples, albeit far lower than what observed for W9, and reverts to zero at RT, which corresponds to completely magnetically unblocked states (Table SI 2). The low values of $H_{\mathrm{E}}^{\mathrm{SEB}}$ in comparison with W9 indicate that the structural rearrangement due to the formation of the $\mathrm{M}_{x} \mathrm{Fe}_{3-x} \mathrm{O}_{4}$ shells $(\mathrm{M}=\mathrm{Co}, \mathrm{Mn})$ is less disordered than the superficial $\mathrm{FeO}$-to$\mathrm{Fe}_{3} \mathrm{O}_{4}$ oxidation of the pristine wüstite sample, thus the "probing field" of the ZFC hysteresis is less likely to "fix" the orientation of spins at the interface. On the other hand, FC hysteresis loops maximize the EB effects by pinning all the uncompensated spins and consequently maximizing the asymmetry of the hysteresis loops. Even under these conditions there is a moderate ${H_{\mathrm{E}}}_{\mathrm{CEB}}$ for W9 $\mathrm{Mn}$ and a strong ${H_{\mathrm{E}}}^{\mathrm{CEB}}$ for W9_Co, although both are far smaller than W9. This confirms that final effect of $\mathrm{CE}$ reactions is the formation of magnetically distinct shells with limited structural disorder, i.e., with a limited amount of uncompensated spins, together with a general magnetic softening/hardening commanded by the magnetic features of the thus formed FiM shells. The formation of well-ordered Co- $[\mathrm{Mn}-] \mathrm{Fe}_{2} \mathrm{O}_{4}$ shells in W9_Co and W9_Mn also implies the minimization of cationic vacancies at the surface of the NPs, which is expected to stabilize the AFM wüstite constituting the core and preventing its oxidation over time. ZFC and FC hysteresis loops were repeated after leaving the samples in air for over six months to verify the possible oxidation of the cores and showed a perfect match with the original curves (Figure SI_10), thus proving the insulating capabilities of the ferrite shell.

Despite all the differences above-reported between the M10based and the W9-based samples, even for the latter ones, a clear vertical shift was observed in all the FC hysteresis loops. As well, this shift could be ascribed to a spin-glass-like magnetic behavior, occurring in a similar fashion to what reported in ref 47 and observed for the M10-based samples, i.e., due to their superficial spins. Also, CE shows once again a non-negligible but partial capability in curing the spin superficial disorder, as indicated by the reduction of the loops vertical shift when passing from W9 to W9_Co and W9_Mn samples. Thus, even if a complete spin-glass-like magnetic behavior characterization is far beyond the main aim of this manuscript, we studied the time dependence of remanent magnetization at low temperature, which is known to reveal the possible spin-glass-like nature of an ensemble of magnetic nanoparticles, as extensively reported in literature. $^{58-61}$ All the thermo-remanent magnetization's time dependences were fitted by using the well-known stretchedexponential law:

$$
M(t)=M_{2}+\left(M_{1}-M_{2}\right) \exp \left(-(t / \tau)^{\beta}\right)
$$

where $M_{1}$ and $M_{2}$ are the initial and final magnetization, respectively after the magnetic field has been turned off, and $\beta$ the so-called critical exponent. Literature reports that below the spin-glass transition temperature, $\beta$ is expected to fall in the range $0.2-0.6 .^{60,61}$ Indeed, this is what we found for all samples (as shown by the fit of the curves and $\beta$ values reported in Figure SI_11), thus confirming that all our systems present spin-glass-like features.

\section{CONCLUSIONS}

Despite the seemingly potential effectiveness of $\mathrm{CE}$ for synthesizing NPs with subtly regulated magnetic features, recent literature reported very few cases where $\mathrm{CE}$ was actually used with that aim and with quite debatable results. ${ }^{18,19}$ Thus, in order to show if $\mathrm{CE}$ has some intrinsic, hidden limitation when applied to magnetic NPs constituted by transition metal oxides, we applied it to starting NPs of two different iron oxide phases, magnetite $\left(\mathrm{Fe}_{3} \mathrm{O}_{4}\right)$ and wüstite $\left(\mathrm{Fe}_{x} \mathrm{O}\right)$. Two NPs populations with the same diameter and spherical shape were prepared with the aim to substitute the iron cations with either cobalt or manganese ones upon previously tested safe conditions, which were not capable of modifying size and shape of the starting NPs. We then performed morphological, structural, and spatially resolved chemical characterization by advanced electron microscopy of both the initial and cationexchanged NPs. Additionally, their magnetic behavior was studied in-depth by SQUID magnetometry. On one hand, we found that under the conditions we used, CE unavoidably leads to core/shell systems. On the other hand, we uncovered the fundamental role played by the crystal structure of the two iron oxide's initial phases (magnetite and wüstite) in the final outcome of the CE approach. In fact, the percentage of exchanging cations (Co or $\mathrm{Mn}$ ) that substituted the iron ones was much higher starting from an intrinsically defective iron oxide crystal phase (wüstite) than starting from undefective magnetite, thus highlighting the role of the vacancies in the effectiveness of the CE. In turn, this gave rise to dramatic 
effects in terms of magnetic behavior of the final NPs, which showed the typical features of magnetic core/shell nanostructures, also strongly dependent on both kind and amount of the cations that replace the iron ones. In the light of our results, we also conducted a deep revision of the few papers published on CE applied to metal oxide NPs, aiming to explain the points still debatable and to provide some general insights about the $\mathrm{CE}$ effectiveness as a synthetic strategy to finely tune the properties of this kind of magnetic NPs, while also showing the occurrence of spin-glass-like magnetic behavior in the low thermal range.

\section{ASSOCIATED CONTENT}

\section{S Supporting Information}

The Supporting Information is available free of charge on the ACS Publications website at DOI: 10.1021/acs.chemmater.8b04331.

STEM/EDS maps of samples M21_Co and M21_Mn. TEM images, STEM/EDS maps and corresponding compositional profiles of samples M21_Co_hc, M10_Mn_hc1 and M10_Mn_hc2. STEM/ED̄S maps of samples M10_Co and $\bar{M} 10 \_\bar{M}$. XRD patterns of the W9 sample in air. STEM/EDS maps of samples W9 Co and W9_Mn. Hysteresis loops of samples $\bar{M} 10$, M10_Co, M10_Mn, W9, W9 Co and W9 Mn recorded at $4 \mathrm{~K}$ and RT. TEM images, STEM/EDS map and corresponding compositional profile of sample W9ox_Mn. ZFC-FC magnetization curves and hysteresis loop of the fully oxidized sample W9. ZFC-FC hysteresis loops of samples W9_Co and W9_Mn. Magnetization relaxation curves of samples $\bar{M} 10$, M10 Co, M10 Mn, W9, W9 Co and W9 Mn. Tables with parameters of hysteresis loops at RT for magnetite and wüstite-based samples (PDF)

\section{AUTHOR INFORMATION}

\section{Corresponding Authors}

*E-mail alberto.casu@kaust.edu.sa (A.C.).

*E-mail andrea.falqui@kaust.edu.sa (A.F.).

\section{ORCID $\odot$}

Elisa Sogne: 0000-0003-2097-4358

Andrea Falqui: 0000-0002-1476-7742

\section{Author Contributions}

A.F. and A.C. conceived the entire project, participated in all experimental phases, and wrote the paper. S.L-M. synthesized all the materials, performed the XRD measurements and contributed to the paper's writing. D.D. performed most of the TEM/STEM-EDS imaging and the corresponding data analysis. E.S. performed several checks of all sample composition by scanning electron microscopy (SEM) (results not shown). All the authors read, commented, and approved the manuscript.

\section{Funding}

We acknowledge financial support from the King Abdullah University of Science and Technology (KAUST) start-up and baseline funding from Professor Andrea Falqui.

\section{Notes}

The authors declare no competing financial interest.

\section{ACKNOWLEDGMENTS}

Table of contents graphic was produced by Heno Hwang, scientific illustrator at KAUST, who is gratefully acknowledged. Mr. Efisio Zuddas, who participated to the preparation of some samples, is also thankfully acknowledged.

\section{ABBREVIATIONS}

$\mathrm{CE}$, cation exchange; NPs, nanoparticles; XRD, X-ray power transmission; TEM, transmission electron microscopy; STEM, scanning transmission electron microscopy; EDS, energydispersive X-ray spectroscopy; EB, exchange-bias; ZFC, zerofield-cooled; FC, field-cooled; $T_{\mathrm{B}}$, blocking temperature; $T_{\text {IRR }}$ irreversibility temperature; $M_{S}$, saturation magnetization; fcc, face centered cubic; AFM, antiferromagnetic; FiM, ferrimagnetic; $M_{R}$ remanence magnetization; SEB, spontaneous exchange bias; $\mathrm{CEB}$, conventional exchange bias; PM, paramagnetic.

\section{REFERENCES}

(1) Lu, A.-H.; Salabas, E. L.; Schüth. Magnetic Nanoparticles: Synthesis, Protection, Functionalization, and Application. Angew. Chem., Int. Ed. 2007, 46, 1222-1244.

(2) Lee, N.; Yoo, D.; Ling, D.; Cho, M. H.; Hyeon, T.; Cheon, J. Iron Oxide Based Nanoparticles for Multimodal Imaging and Magnetoresponsive Therapy. Chem. Rev. 2015, 115, 10637-10689.

(3) Frey, N. A.; Peng, S.; Cheng, K.; Sun, S. Magnetic Nanoparticles: Synthesis, Functionalization, and Applications in Bioimaging and Magnetic Energy Storage. Chem. Soc. Rev. 2009, 38, 2532-2542.

(4) Laurent, S.; Forge, D.; Port, M.; Roch, A.; Robic, C.; Elst, L. V.; Muller, R. N. Magnetic Iron Oxide Nanoparticles: Synthesis, Stabilization, Vectorization, Physicochemical Characterizations, and Biological Applications. Chem. Rev. 2008, 108, 2064-2110.

(5) Yang, C.; Wu, J.; Hou, Y. $\mathrm{Fe}_{3} \mathrm{O}_{4}$ Nanostructures: Synthesis, Growth Mechanism, Properties and Applications. Chem. Commun. 2011, 47, 5130-5141.

(6) Rivest, J. B.; Jain, P. K. Cation Exchange on the Nanoscale: an Emerging Technique for New Material Synthesis, Device Fabrication, and Chemical Sensing. Chem. Soc. Rev. 2013, 42, 89-96.

(7) Beberwyck, B. J.; Surendranath, Y.; Alivisatos, P. Cation Exchange: a Versatile Tool for Nanomaterials Synthesis. J. Phys. Chem. C 2013, 117, 19759-19770.

(8) Hodges, J. M.; Kletetschka, K.; Fenton, J. L.; Read, C. G.; Schaak, R. E. Sequential Anion and Cation Exchange Reactions for Complete Material Transformations of Nanoparticles with Morphological Retention. Angew. Chem., Int. Ed. 2015, 54, 8669-8672.

(9) Beberwyck, B. J.; Alivisatos, A.-P. Ion Exchange Synthesis of IIIV Nanocrystals. J. Am. Chem. Soc. 2012, 134, 19977-19980.

(10) Ha, D. H.; Caldwell, A. H.; Ward, M. J.; Honrao, S.; Mathew, K.; Hovden, R.; Koker, M. K.; Muller, D. A.; Hennig, R. G.; Robinson, R. D. Solid-Solid Phase Transformations Induced through Cation Exchange and Strain in 2D Heterostructured Copper Sulfide Nanocrystals. Nano Lett. 2014, 14, 7090-7099.

(11) Justo, Y.; Sagar, L. K.; Flamee, S.; Zhao, Q.; Vantomme, A.; Hens, Z. Chemistry of the Nanocrystal Surface. ACS Nano 2014, 8, $7948-7957$

(12) Casavola, M.; van Huis, M. A.; Bals, S.; Lambert, K.; Hens, Z.; Vanmaekelbergh, D. Anisotropic Cation Exchange in $\mathrm{PbSe} / \mathrm{CdSe}$ Core/Shell Nanocrystals of Different Geometry. Chem. Mater. 2012, 24, 294-302.

(13) De Trizio, L.; Manna, L. Forging Colloidal Nanostructures via Cation Exchange Reactions. Chem. Rev. 2016, 116, 10852-10887.

(14) De Trizio, L.; De Donato, F.; Casu, A.; Genovese, A.; Falqui, A.; Povia, M.; Manna, L. Colloidal CdSe $/ \mathrm{Cu}_{3} \mathrm{P} / \mathrm{CdSe}$ Nanocrystal Heterostructures and their Evolution upon Thermal Annealing. ACS Nano 2013, 7, 3997-4005.

(15) De Trizio, L.; Figuerola, A.; Manna, L.; Genovese, A.; George, C.; Brescia, R.; Saghi, Z.; Simonutti, R.; Van Huis, M.; Falqui, A. Size- 
Tunable, Hexagonal Plate-Like $\mathrm{Cu}_{3} \mathrm{P}$ and Janus-Like $\mathrm{Cu}_{3} \mathrm{P}-\mathrm{Cu}$ Nanocrystals. ACS Nano 2012, 6, 32-41.

(16) Casu, A.; Genovese, A.; Manna, L.; Longo, P.; Buha, J.; Botton, G. A.; Lazar, S.; Kahaly, M. U.; Schwingenschloegl, U.; Prato, M.; Li, H.; Ghosh, S.; Palazon, F.; De Donato, F.; Lentijo-Mozo, S.; Zuddas, E.; Falqui, A. $\mathrm{Cu}_{2} \mathrm{Se}$ and $\mathrm{Cu}$ Nanocrystals as Local Sources of Copper in Thermally Activated In Situ Cation Exchange. ACS Nano 2016, 10, 2406-2414.

(17) Lesnyak, V.; Brescia, R.; Messina, G. C.; Manna, L. Cu Vacancies Boost Cation Exchange Reactions in Copper Selenide Nanocrystals. J. Am. Chem. Soc. 2015, 137, 9315-9323.

(18) Sytnyk, M.; Kirchschlager, R.; Bodnarchuk, M. I.; Primetzhofer, D.; Kriegner, D.; Enser, H.; Stangl, J.; Bauer, P.; Voith, M.; Hassel, A.; et al. Tuning the Magnetic Properties of Metal-Oxide Nanocrystal Heterostructures by Cation Exchange. Nano Lett. 2013, 13, 586-593.

(19) Zhao, Z.; Chi, X.; Yang, L.; Yang, R.; Ren, B. W.; Zhu, X.; Zhang, P.; Gao, J. Cation Exchange of Anisotropic-Shaped Magnetite Nanoparticle Generates High-Relaxivity Contrast Agents for Liver 937 Tumor Imaging. Chem. Mater. 2016, 28, 3497-3506.

(20) Kim, Y. I.; Kim, D.; Lee, C. S. Synthesis and Characterization of $\mathrm{CoFe}_{2} \mathrm{O}_{4}$ Magnetic Nanoparticles Prepared by Temperature-Controlled Coprecipitation Method. Phys. B 2003, 337, 42-51.

(21) Goya, G. F.; Berquó, T. S.; Fonseca, F. C.; Morales, M. P. Static and Dynamic Magnetic Properties of Spherical Magnetite Nanoparticles. J. Appl. Phys. 2003, 94, 3520-3528.

(22) Brabers, V. A. M. In Handbook of Magnetic Materials; Buschow, K. H. J., Ed.; North-Holland: Amsterdam, 1995; Vol. 8, p 212.

(23) Nogués, J.; Sort, J.; Langlais, V.; Skumryev, V.; Suriñach, S.; Muñoz, J. S.; Baró, M. D. Exchange Bias in Nanostructures. Phys. Rep. 2005, 422, 65-117.

(24) Sahoo, R. C.; Paladhi, D.; Dasgupta, P.; Poddar, A.; Singh, R.; Das, A.; Nath, T. K. Antisite-Disorder Driven Large Exchange Bias Effect in Phase Separated $\mathrm{La}_{1.5} \mathrm{Ca}_{0.5} \mathrm{CoMnO}_{6}$ Double Perovskite. J. Magn. Magn. Mater. 2017, 428, 86-91.

(25) Luo, Z.; Irtem, E.; Ibanez, M.; Nafria, R.; Marti-Sanchez, S.; Genc, A.; De la Mata, M.; Liu, Y.; Cadavid, D.; Llorca, J.; et al. $\mathrm{Mn}_{3} \mathrm{O}_{4} @ \mathrm{CoMn}_{2} \mathrm{O}_{4}-\mathrm{Co}_{\mathrm{x}} \mathrm{O}_{\mathrm{y}}$. Nanoparticles: Partial Cation Exchange Synthesis and Electrocatalytic Properties toward the Oxygen Reduction and Evolution Reactions. ACS Appl. Mater. Interfaces 2016, 8, 17435-17444.

(26) Zuddas, E.; Lentijo-Mozo, S.; Casu, A.; Deiana, D.; Falqui, A. Building Composite Iron-Manganese Oxide Flowerlike Nanostructures: a Detailed Magnetic Study. J. Phys. Chem. C 2017, 121, 1700517015 .

(27) Park, J.; An, K.; Hwang, Y.; Park, J. G.; Noh, H. J.; Kim, K. Y.; Park, J. H.; Hwang, N. M.; Hyeon, T. Ultra-Large-Scale Syntheses of Monodisperse Nanocrystals. Nat. Mater. 2004, 3, 891-895.

(28) Ghosh Chaudhuri, R.; Paria, S. Core/Shell Nanoparticles: Classes, Properties, Synthesis, Mechanisms, Characterization, and Applications. Chem. Rev. 2012, 112, 2373-2433.

(29) Mazumder, V.; Chi, M.; More, K. L.; Sun, S. Synthesis and Characterization of Multimetallic $\mathrm{Pd} / \mathrm{Au}$ and $\mathrm{Pd} / \mathrm{Au} / \mathrm{FePt}$ Core/Shell Nanoparticles. Angew. Chem., Int. Ed. 2010, 49, 9368-9372.

(30) Grouchko, M.; Kamyshny; Magdassi, S. Formation of AirStable Copper-Silver Core-Shell Nanoparticles for Inkjet Printing. J. Mater. Chem. 2009, 19, 3057-3062.

(31) Wang, J. X.; Inada, H.; Wu, L.; Zhu, Y.; Choi, Y.; Liu, P.; Zhou, W.-P.; Adzic, R. R. Oxygen Reduction on Well-Defined Core-Shell Nanocatalysts: Particle Size, Facet, and Pt Shell Thickness Effects. J. Am. Chem. Soc. 2009, 131, 17298-17302.

(32) Baaziz, W.; Pichon, P. P.; Fleutot, S.; Liu, Y.; Lefevre, C.; Greneche, J. M.; Toumi, M.; Mhiri, T.; Begin-Colin, S. Magnetic Iron Oxide Nanoparticles: Reproducible Tuning of the Size and Nanosized-Dependent Composition, Defects, and Spin Canting. J. Phys. Chem. C 2014, 118, 3795-3810.

(33) Wu, W.; Wu, Z.; Yu, T.; Jiang, C.; Kim, W.-S. Recent Progress on Magnetic Iron Oxide Nanoparticles: Synthesis, Surface Functional Strategies and Biomedical Applications. Sci. Technol. Adv. Mater. 2015, 16,023501 .
(34) Luo, Z.; Marti-Sanchez, S.; Nafria, R.; Joshua, G.; de la Mata, M.; Guardia, P.; Flox, C.; Martinez-Boubeta, C.; Simeonidis, K.; Llorca, J.; Morante, J.-R.; Arbiol, J.; Ibanez, M.; Cabot, A. $\mathrm{Fe}_{3} \mathrm{O}_{4} @$ $\mathrm{NiFe}_{\mathrm{x}} \mathrm{O}_{\mathrm{y}}$ Nanoparticles with Enhanced Electrocatalytic Properties for Oxygen Evolution in Carbonate Electrolyte. ACS Appl. Mater. Interfaces 2016, 8, 29461-29469.

(35) Khurshid, H.; Li, W.; Chandra, S.; Phan, M. H.; Hadjipanayis, G.; Mukherjee, P.; Srikanth, H. Mechanism and Controlled Growth of Shape and Size Variant Core/Shell $\mathrm{FeO} / \mathrm{Fe}_{3} \mathrm{O}_{4}$ Nanoparticles. Nanoscale 2013, 5, 7942-7952.

(36) Chen, C. J.; Chiang, R. K.; Kamali, S.; Wang, S. L. Synthesis and Controllable Oxidation of Monodisperse Cobalt-Doped Wüstite Nanoparticles and their Core-Shell Stability and Exchange-Bias Stabilization. Nanoscale 2015, 7, 14332-14343.

(37) Hou, Y.; Xu, Z.; Sun, S. Controlled Synthesis and Chemical Conversions of $\mathrm{FeO}$ Nanoparticles. Angew. Chem., Int. Ed. 2007, 46, 6329-6332.

(38) Hufschmid, R.; Arami, H.; Matthew Ferguson, R.; Gonzales, M.; Teeman, E.; Brush, L. N.; Browning, N. D.; Krishnan, K. M. Synthesis of Phase-Pure and Monodisperse Iron Oxide Nanoparticles by Thermal Decomposition. Nanoscale 2015, 7, 11142-11154.

(39) Fan, Z.; Lin, L.-C.; Buijs, W.; Vlugt, T. J. H.; van Huis, M. A. Atomistic Understanding of Cation Exchange in $\mathrm{PbS}$ Nanocrystals Using Simulations with Pseudoligands. Nat. Commun. 2016, 7, 11503.

(40) Bodnarchuk, M. I.; Kovalenko, M. V.; Groiss, H.; Resel, R.; Reissner, M.; Hesser, G.; Lechner, R. T.; Steiner, W.; Schäffler, F.; Heiss, W. Exchange-Coupled Bimagnetic Wüstite/Metal Ferrite Core/Shell Nanocrystals: Size, Shape and Compositional Control. Small 2009, 5, 2247-2252.

(41) Cornell, R. M.; Schwertmann, U. The Iron Oxides: Structure, Properties, Reactions, Occurrences and Uses; Wiley-VCH: Weinheim, Germany, 2003.

(42) Balaji, G.; Gajbhiye, N. S.; Wilde, G.; Weissmuller, J. Magnetic Properties of $\mathrm{MnFe}_{2} \mathrm{O}_{4}$ Nanoparticles. J. Magn. Magn. Mater. 2002, 242-245, 617-620.

(43) Brabers, V. A. Progress in Spinel Ferrite Research. In Handbook of Magnetic Materials; Buschow, K. H. J., Ed.; North-Holland: Amsterdam, The Netherlands, 1995; Vol. 8

(44) Wan, J.; Tang, G.; Qian, Y. Room Temperature Synthesis of Single-Crystal $\mathrm{Fe}_{3} \mathrm{O}_{4}$ Nanoparticles with Superparamagnetic Property. Appl. Phys. A: Mater. Sci. Process. 2006, 86, 261-264.

(45) Yoon, S.; Krishnan, K. M. Temperature Dependence of Magnetic Anisotropy Constant in Manganese Ferrite Nanoparticles at Low Temperature. J. Appl. Phys. 2011, 109, 07B534.

(46) Lima, E.; Brandl, A. L.; Arelaro, A. D.; Goya, G. F. Spin Disorder and Magnetic Anisotropy in $\mathrm{Fe}_{3} \mathrm{O}_{4}$ Nanoparticles. J. Appl. Phys. 2006, 99, 083908.

(47) Khurshid, H.; Lampen-Kelley, P.; Iglesias, O.; Alonso, J.; Phan, M.-H.; Sun, C.-J.; Saboungi, M.-L.; Srikanth, H. Spin-Glass-Like Freezing of Inner and Outer Surface Layers in hollow $\gamma-\mathrm{Fe}_{2} \mathrm{O}_{3}$ Nanoparticles. Sci. Rep. 2015, 5, 15054.

(48) Zheng, X. G.; Xu, C. N.; Nishikubo, K.; Nishiyama, K.; Higemoto, W.; Moon, W. J.; Tanaka, E.; Otabe, E. S. Finite-Size Effect on Néel Temperature in Antiferromagnetic Nanoparticles. Phys. Rev. B: Condens. Matter Mater. Phys. 2005, 72, 014464.

(49) Thota, S.; Shim, J. H.; Seehra, M. S. Size-Dependent Shifts of the Néel Temperature and Optical Band-Gap in NiO. Nanoparticles. J. Appl. Phys. 2013, 114, 214307.

(50) Sako, S.; Ohshima, K. Antiferromagnetic Transition Temperature of MnO Ultrafine Particle. J. Phys. Soc. Jpn. 1995, 64, 944-950.

(51) Lottini, E.; López-Ortega, A.; Bertoni, G.; Turner, S.; Meledina, M.; Van Tendeloo, G.; de Julián Fernández, C.; Sangregorio, C. Strongly Exchange Coupled CorelShell Nanoparticles with High Magnetic Anisotropy: A Strategy toward Rare-Earth-Free Permanent Magnets. Chem. Mater. 2016, 28, 4214-4222.

(52) Estrader, M.; López-Ortega, A.; Golosovsky, I. V.; Estradé, S.; Roca, A. G.; Salazar-Alvarez, G.; López-Conesa, L.; Tobia, D.; Winkler, E.; Ardisson, J. D.; Macedo, W. A. A.; Morphis, A.; Vasilakaki, M.; Trohidou, K. N.; Gukasov, A.; Mirebeau, I.; Makarova, 
O. L.; Zysler, R. D.; Peiró, F.; Baró, M. D.; Bergström, L.; Nogués, J. Origin of the Large Dispersion of Magnetic Properties in Nanostructured Oxides: $\mathrm{Fe}_{\mathrm{x}} \mathrm{O} / \mathrm{Fe}_{3} \mathrm{O}_{4}$ Nanoparticles as a Case Study. Nanoscale 2015, 7, 3002-3015.

(53) Gheisari, M.; Mozaffari, M.; Acet, M.; Amighian, J. Preparation and Investigation of Magnetic Properties of Wüstite Nanoparticles. J. Magn. Magn. Mater. 2008, 320, 2618-2621.

(54) Pichon, B. P.; Gerber, O.; Lefevre, C.; Florea, I.; Fleutot, S.; Baaziz, W.; Pauly, M.; Ohlmann, M.; Ulhaq, C.; Ersen, O.; PierronBohnes, V.; Panissod, P.; Drillon, M.; Begin-Colin, S. Microstructural and Magnetic Investigations of Wüstite-Spinel Core-Shell CubicShaped Nanoparticles. Chem. Mater. 2011, 23, 2886-2900.

(55) Leszczyński, B.; Hadjipanayis, G. C.; El-Gendy, A. A.; Załęski, K.; Sniadecki, Z.; Musial, A.; Jarek, M.; Jurga, S.; Skumiel, A. The Influence of Oxidation Process on Exchange Bias in Egg-Shaped $\mathrm{FeO} /$ $\mathrm{Fe}_{3} \mathrm{O}_{4}$ Core/Shell Nanoparticles. J. Magn. Magn. Mater. 2016, 416, 269-274.

(56) Gheisari, M.; Mozafari, M.; Niyaifar, M.; Amighian, J.; Soleimani, R. Observation of Small Exchange Bias in Defect Wüstite $\left(\mathrm{Fe}_{0.93} \mathrm{O}\right)$ Nanoparticles. J. Supercond. Novel Magn. 2013, 26, 237242.

(57) Sharma, S. K.; Vargas, J. M.; Pirota, K. R.; Kumar, S.; Lee, C. G.; Knobel, M. Synthesis and Ageing Effect in $\mathrm{FeO}$ Nanoparticles: Transformation to Core-Shell $\mathrm{FeO} / \mathrm{Fe}_{3} \mathrm{O}_{4}$ and their Magnetic Characterization. J. Alloys Compd. 2011, 509, 6414-6417.

(58) Dutta, P.; Pramanick, S.; Venkateshwarlu, D.; Ganesan, V.; Majumdar, S.; Das, D.; Chatterjee, S. Spin-Glass-Like Ground State and Observation of Exchange Bias in $\mathrm{Mn}_{0.8} \mathrm{Fe}_{0.2} \mathrm{NiGe}$ Alloy. EPL 2014, 108, 17012.

(59) Falqui, A.; Lampis, N.; Geddo-Lehmann, A.; Pinna, G. J. LowTemperature Magnetic Behavior of Perovskite Compounds $\mathrm{PbFe}_{1 / 2} \mathrm{Ta}_{1 / 2} \mathrm{O}_{3}$. J. Phys. Chem. B 2005, 109, 22967-22970.

(60) Zeb, F.; Nadeem, K.; Ali Shah, S. K.; Kamran, M.; Gul, I. H.; Ali, L. Surface Spins Disorder in Uncoated and $\mathrm{SiO}_{2}$ Coated Maghemite Nanoparticles. J. Magn. Magn. Mater. 2017, 429, 270275.

(61) Sasaki, M.; Jonsson, P. E.; Takayama, H.; Mamiya, H. Aging and Memory Effects in Superparamagnets and Superspin glasses. Phys. Rev. B: Condens. Matter Mater. Phys. 2005, 71, 104405. 